\title{
Inhibiting eukaryotic ribosome biogenesis
}

\author{
Dominik Awad ${ }^{1,2}$, Michael Prattes ${ }^{1}$, Lisa Kofler ${ }^{1}$, Ingrid Rössler ${ }^{1}$, Mathias Loibl ${ }^{1}$, Melanie Pertl ${ }^{1}$, Gertrude Zisser ${ }^{1}$, \\ Heimo Wolinski ${ }^{1}$, Brigitte Pertschy ${ }^{1 *}$ and Helmut Bergler ${ }^{1 *}$ (i)
}

\begin{abstract}
Background: Ribosome biogenesis is a central process in every growing cell. In eukaryotes, it requires more than 250 non-ribosomal assembly factors, most of which are essential. Despite this large repertoire of potential targets, only very few chemical inhibitors of ribosome biogenesis are known so far. Such inhibitors are valuable tools to study this highly dynamic process and elucidate mechanistic details of individual maturation steps. Moreover, ribosome biogenesis is of particular importance for fast proliferating cells, suggesting its inhibition could be a valid strategy for treatment of tumors or infections.

Results: We systematically screened 1000 substances for inhibitory effects on ribosome biogenesis using a microscopybased screen scoring ribosomal subunit export defects. We identified 128 compounds inhibiting maturation of either the small or the large ribosomal subunit or both. Northern blot analysis demonstrates that these inhibitors cause a broad spectrum of different rRNA processing defects.

Conclusions: Our findings show that the individual inhibitors affect a wide range of different maturation steps within the ribosome biogenesis pathway. Our results provide for the first time a comprehensive set of inhibitors to study ribosome biogenesis by chemical inhibition of individual maturation steps and establish the process as promising druggable pathway for chemical intervention.
\end{abstract}

\section{Background}

Ribosomes are essential nano-machines responsible for the synthesis of proteins. They are composed of a large and a small subunit, both containing ribosomal RNAs (rRNAs) and numerous ribosomal proteins. In eukaryotes, the formation of ribosomes is a complex, multi-compartmental process requiring a multitude of non-ribosomal assembly factors. Ribosome biogenesis is highly conserved among eukaryotes and best studied in the yeast Saccharomyces cerevisiae (reviewed in [1-4]). The initial steps of ribosome biogenesis take place in the nucleolus, a sub-compartment of the nucleus, in which the rRNA precursors are transcribed and loaded with assembly factors and ribosomal proteins. The small 5S rRNA of the large 60S subunit is transcribed separately by RNA polymerase III, while the $18 \mathrm{~S}$ rRNA, constituent of the small $40 \mathrm{~S}$ subunit, and the $25 \mathrm{~S}$ and $5.8 \mathrm{~S}$ rRNAs of the large subunit are transcribed together by RNA polymerase I in a polycistronic $35 \mathrm{~S}$ transcript. This long pre-rRNA is co-transcriptionally

\footnotetext{
* Correspondence: brigitte.pertschy@uni-graz.at; helmut.bergler@uni-graz.at ${ }^{1}$ Institute of Molecular Biosciences, University of Graz, Humboldtstrasse 50/ EG, A-8010 Graz, Austria

Full list of author information is available at the end of the article
}

recognized by a plethora of small subunit assembly factors forming a large $90 \mathrm{~S}$ ribosomal precursor also termed the small subunit (SSU) processome $([5,6]$ reviewed in [7]). After stepwise truncation at the $5^{\prime}$-end by endonucleases, cleavage at site $\mathrm{A}_{2}$ leads to $20 \mathrm{~S}$ and $27 \mathrm{SA}_{2}$ pre-rRNAs, thereby separating small and large subunit assembly into independent pathways. The resulting pre-40S particles containing the 20S pre-rRNA are quickly exported into the cytoplasm, where the final maturation steps are accomplished by an endonucleolytic cleavage step at the 3 '-end of the 18S rRNA (for a recent review of $40 \mathrm{~S}$ assembly, see [8]). The process of pre-60S maturation is more complex, involving stepwise endo- and exonucleolytic $5^{\prime}$-end truncations of the $27 \mathrm{SA}_{2}$ pre-rRNA into the $27 \mathrm{SA}_{3}$ and the 27SB pre-rRNA (for a recent review of $60 \mathrm{~S}$ assembly, see [9]). The 27SB pre-rRNA is subsequently split by endonucleolytic cleavage into a $5.8 \mathrm{~S}$ precursor $(7 \mathrm{~S}$ prerRNA) and a $25 \mathrm{~S}$ precursor (25.5S pre-rRNA). In the course of these maturation steps, pre-60S particles transit from the nucleolus to the nucleoplasm. While the mature $25 \mathrm{~S}$ rRNA is finalized by $5^{\prime}-3^{\prime}$ exonucleases in the nucleoplasm, processing of the $7 \mathrm{~S}$ to the $5.8 \mathrm{~S}$ rRNA occurs in several $3{ }^{\prime}-5^{\prime}$ exonucleolytic steps, first in the nucleoplasm 
and then, after nuclear export of the pre-60S particles, in the cytoplasm (see Additional file 1: Figure S1 for a schematic depiction of rRNA processing and $[10,11]$ for comprehensive reviews). Transport of export competent particles of both subunits through the nuclear pore complex depends on the Ran-GTP-dependent exportin Crm1 (XpoI) as well as specialized export factors like Mex67/ Mtr2 or Arx1 [12, 13].

Along with the rRNA processing steps, pre-ribosomal particles undergo massive structural re-arrangements, as impressively evidenced by several recently published highresolution cryo-electron microscopic structures of $90 \mathrm{~S}$, pre$60 \mathrm{~S}$, and pre-40S particles representing different maturation stages [14-21].

All these maturation steps are performed by more than 250 , mostly essential assembly factors representing a wide variety of functions. Considering the number of involved factors, as well as the expenses for rRNA transcription and ribosomal protein synthesis, ribosome biogenesis represents a major activity in each cell [22]. Therefore, it is of particular importance for fast dividing cells and tightly linked to cell division and cell cycle progression. All these facts make ribosome biogenesis an exceptionally promising target for chemotherapeutical intervention during infectious with eukaryotic pathogens or neoplastic diseases [23-26]. Indeed, several established chemotherapeutic drugs were shown to also inhibit ribosome biogenesis [24], suggesting that this pathway is either their primary target or a second site target whose inhibition enhances the potential of chemotherapeutical agents. In both scenarios, inhibition of ribosome biogenesis can contribute to the anti-proliferative effect of chemotherapy.

While several inhibitors of rRNA transcription have been reported [23, 26], only very few inhibitors are known that directly target the ribosome biogenesis pathway downstream of transcription. We previously discovered that the drug diazaborine specifically inhibits large ribosomal subunit formation by preventing the cytoplasmic release of the shuttling pre-60S assembly factor Rlp24 by the AAA-ATPase Drg1 [27-29]. This release reaction is a prerequisite for all downstream maturation steps. Consequently, diazaborine treatment prevents the release and the recycling of all known shuttling pre-60S assembly factors. This results in depletion of shuttling pre-60S assembly factors in the nucleus, hence causing defects in early pre-60S maturation. Recently, another ribosome biogenesis inhibitor (ribozinoindole) was described, which targets the nuclear AAA-ATPase Mdn1 in Schizosaccharomyces pombe [30]. The homologous protein in S. cerevisiae, Rea1, is required for pre-60S release of the assembly factor Rsa4 and might play a role in a major structural transition of the pre-60S particle during nucleoplasmic maturation steps $[15,31]$.
In order to explore further promising druggable steps of ribosome biogenesis, we developed a microscopy-based screening approach to identify novel ribosome biogenesis inhibitors. In this study, we systematically screened $\sim 1000$ low molecular weight substances for inhibitory effects on the ribosome biogenesis pathway in S. cerevisiae. One hundred twenty-eight of these compounds led to a nuclear accumulation of fluorescently labeled reporter proteins for either the 40S, the 60S, or both ribosomal subunits. Northern blot analyses revealed that the individual substances affect various stages of pre-rRNA processing, suggesting a broad coverage of different targets along the pathway. By introducing a versatile set of novel ribosome biogenesis inhibitors, our results provide a promising starting point to study this essential pathway in depth by chemical inhibition of individual maturation steps. Moreover, our results provide a basis to establish the ribosome biogenesis pathway as target for chemotherapy.

\section{Results}

Screen setup

In order to identify novel inhibitors of the ribosome synthesis pathway, we designed a strategy that allowed us to systematically screen a large quantity of low molecular weight substances for effects on ribosome biogenesis in the yeast $S$. cerevisiae (summarized in Fig. 1). In total, we tested $\sim 1000$ substances from two different compound libraries. The "NIH clinical collection" comprises 446 small molecules that have already been used in human clinical trials (Additional file 2: Table S1). The "Screen-Well Natural Product Library Version 7.4" from Enzo Life Sciences provides 502 small molecules of natural origin (Additional file 2: Table S2). The rationale behind the screen was based on the observation that inhibition of the ribosome biogenesis pathway frequently causes ribosomal subunit export defects [32]. To score for such export defects, yeast strains expressing C-terminal GFP-tag fusions of a ribosomal protein of either the $60 \mathrm{~S}$ or the $40 \mathrm{~S}$ subunit were generated by chromosomal integration at the genomic loci. For the $60 S$ subunit export screen, we used the large ribosomal subunit protein Rpl7 as reporter (uL30 according to a recently proposed new nomenclature [33]). Rpl7 is incorporated into pre-60S particles at an early maturation stage allowing to score for very early defects in $60 \mathrm{~S}$ synthesis [34-39]. As 40S subunit reporter, we selected Rps9 (uS4), an early assembling 40S subunit ribosomal protein $[16,20,40-42]$. As both proteins have two paralogs in yeast, we chose the more abundant variants Rpl7a and Rps9a for GFP-tag fusions.

As a reference for inhibition of $60 \mathrm{~S}$ subunit export, we treated cells with diazaborine, which causes nuclear accumulation of Rpl7a-GFP due to ribosome biogenesis inhibition ( [28], Fig. 2a). As no inhibitor of the 40S biogenesis pathway was available, we aimed at establishing a positive 


\section{a Screen setup \\ Rpl7-GFP \\ for $60 \mathrm{~S}$ \\ subunit

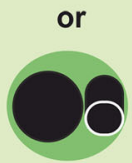 \\ Rps9-GFP \\ for $40 \mathrm{~S}$ \\ subunit \\ ribosomal reporter strain

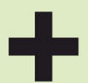 \\ 1000 compounds \\ clinically \\ substances \\ (NIH)

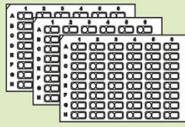 \\ natural \\ products \\ (Enzo)}

\section{b Hit identification}

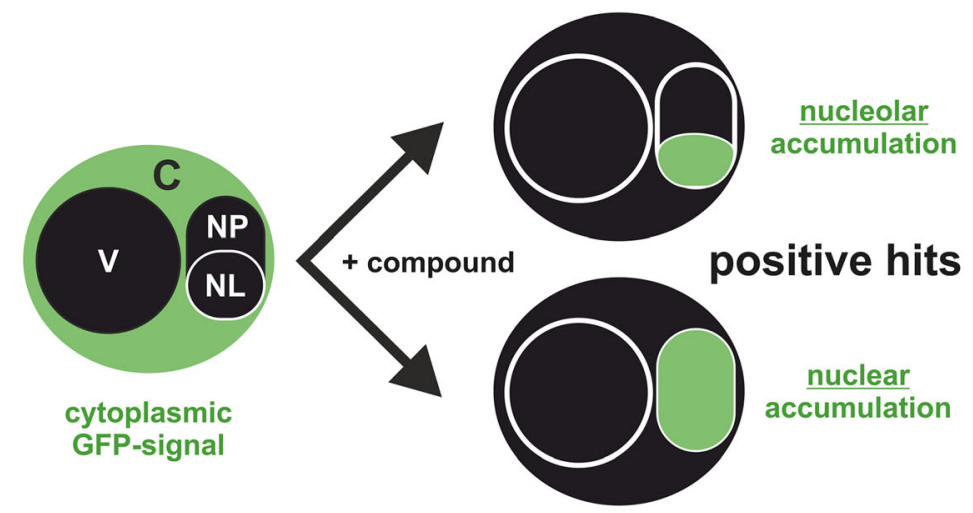

\section{C...Cytoplasm}

\section{V...Vacuole}

NP...Nucleoplasm

NL....Nucleolus

\section{C}

128 potential ribosome biogenesis inhibitors

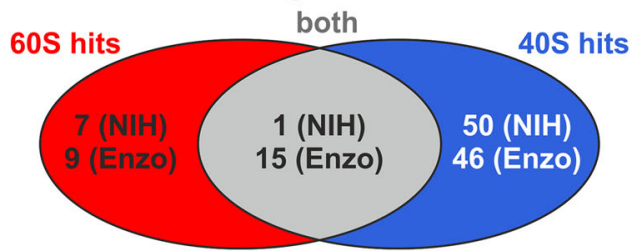

d

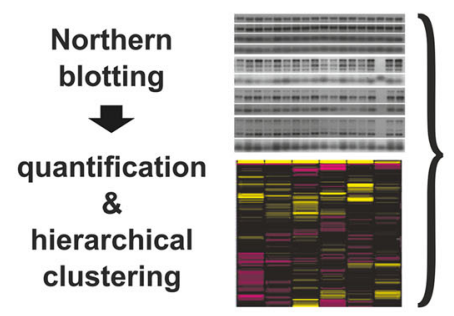

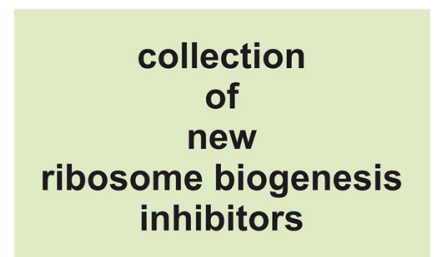

Fig. 1 Screen setup to identify novel ribosome biogenesis inhibitors. a Reporter proteins for either the 40S (Rps9a) or the 60S (Rpl7a) ribosomal subunit were C-terminally fused to GFP. The resulting reporter strains were separately tested with $\sim 1000$ substances from two compound libraries (NIH clinical collection (Additional file 2: Table S1) and Screen-Well Natural Product Library Version 7.4 (Enzo Life sciences) (Additional file 2: Table S2)). b After treatment, cells were inspected by fluorescence microscopy. Ribosome biogenesis defects were identified by a shift of the steady-state GFP signal of the reporters from the cytoplasm into the nucleolus (NL) and/or the nucleoplasm (NP). c A total pool of 128 positively scoring inhibitors comprised 16 inhibitors specific for the 605 subunit, 96 specific for the $40 \mathrm{~S}$ subunit and 16 affecting both subunits. $\mathbf{d}$ Positively scoring hits were further characterized by northern blot analysis of pre-rRNAs and subsequent hierarchical clustering based on quantification of processing intermediates

control causing nuclear accumulation of pre-40S subunits. In the course of the screen with the 60S subunit reporter Rpl7a-GFP, we found that treatment with acivicin resulted in the accumulation of Rpl7a-GFP in small dots in the nucleus which likely correspond to the nucleolus or a sub-fraction thereof (Fig. 2a). This defect is indicative of a very early blockage of ribosome biogenesis.

Since the initial steps of large and small subunit formation are interconnected, we reasoned that early ribosome biogenesis inhibition should affect both the $40 \mathrm{~S}$ and the $60 \mathrm{~S}$ biogenesis pathway. Indeed, acivicin treatment also caused nuclear accumulation of our $40 \mathrm{~S}$ reporter Rps9a-GFP in a dotted structure (Fig. 2b). This demonstrates that the Rps9a-GFP reporter is suitable to detect inhibitor-induced ribosome biogenesis defects and that acivicin can be used as a reference for the $40 \mathrm{~S}$ screen. DMSO alone, the solvent of the tested substances, caused no nuclear accumulation of the ribosomal reporter proteins in tested concentrations up to $6 \%$ (data not shown).

\section{Nucle(ol)ar accumulation of ribosomal subunit reporters identifies 128 potential ribosome biogenesis inhibitors} In the initial large-scale screen, the Rpl7a-GFP and Rps9a-GFP reporter strains were incubated with each of the 948 inhibitors for at least $3 \mathrm{~h}$. Subsequently, the treated cells were inspected by fluorescence microscopy. All substances causing nucle(ol)ar accumulation of one or both reporter constructs were re-analyzed in two additional screening rounds. In total, 128 substances were confirmed as positive hits (Fig. 3 and Additional file 1: Figures S2-S8; see Additional file 3: Table S3 for a complete list of identified substances including references for documented activities against cancer cells [43-113]). 


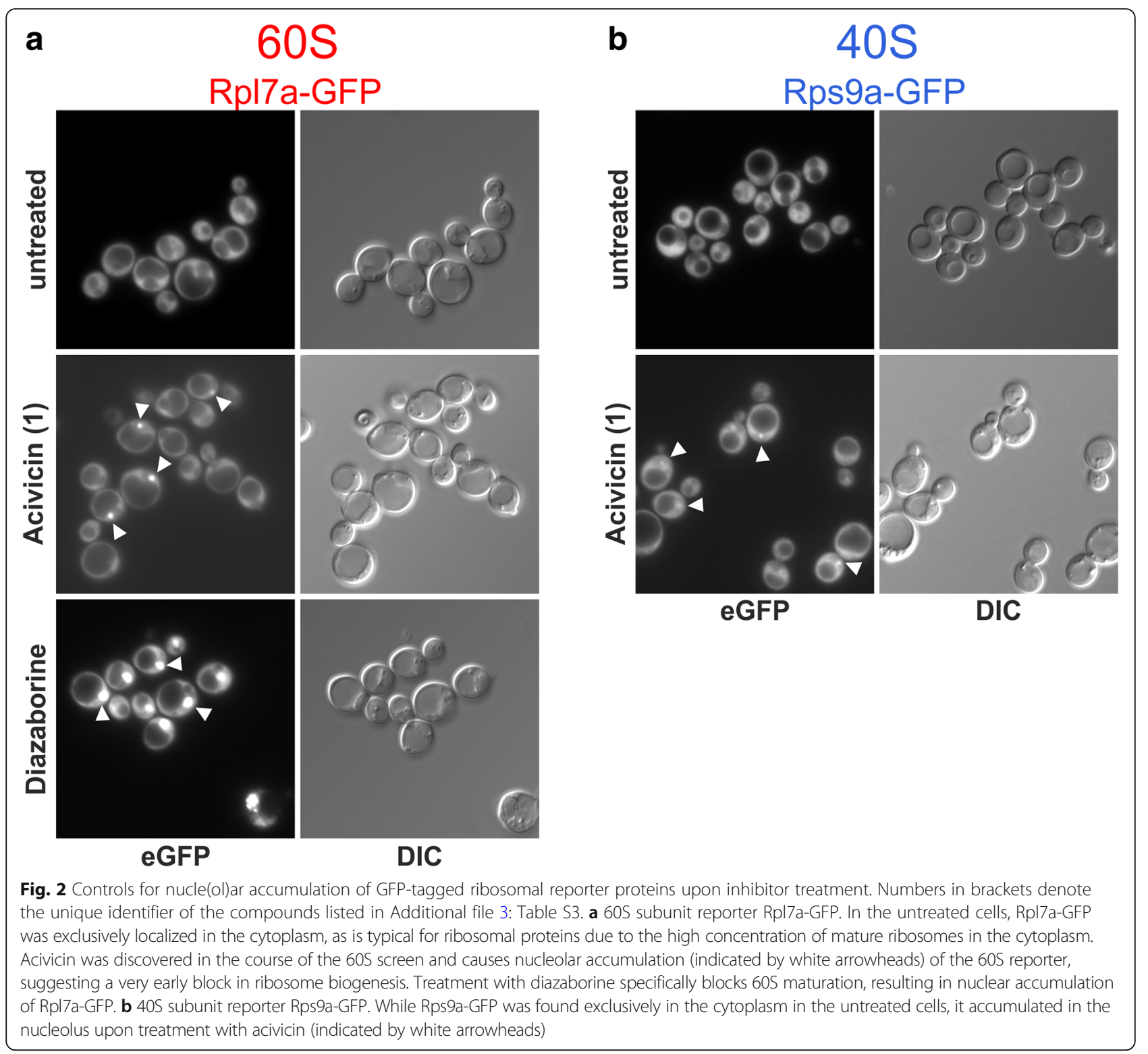

The observed phenotypes included accumulation in the entire nucleolus or smaller dotted structures within the nucleolus (Additional file 1: Figure S9). One of the tested substances from the NIH collection and 15 substances from the Enzo collection caused accumulation of both reporters in the nucleus, suggesting a very early block in ribosome biogenesis (Fig. 3b and Additional file 1: Figure S2). Seven of the NIH compounds and nine of the Enzo compounds caused nuclear accumulation of only the $60 \mathrm{~S}$ subunit reporter Rpl7a-GFP (Fig. 3c and Additional file 1: Figure S3), whereas 50 of the NIH and 46 of the Enzo substances caused nuclear accumulation of the 40S subunit reporter Rps9a-GFP (Fig. 3d and Additional file 1: Figures S4-S8).
To conclude, our microscopy-based screen successfully identified 128 potential ribosome biogenesis inhibitors targeting either the maturation of the small $40 \mathrm{~S}$ subunit, the large 605 subunit, or both.

\section{Inhibitors induce diverse pre-rRNA processing defects}

In order to further validate that the identified substances induce specific ribosome biogenesis defects and to elucidate the approximate stages of inhibition, we investigated their effect on pre-rRNA processing (Fig. 4). For this purpose, wild-type yeast cells were treated with each of the 128 identified substances for $30 \mathrm{~min}$. Subsequently, total RNA was isolated and subjected to northern blotting using probes specifically hybridizing to either mature $25 \mathrm{~S}, 18 \mathrm{~S}, 5.8 \mathrm{~S}$, and $5 \mathrm{~S}$ 


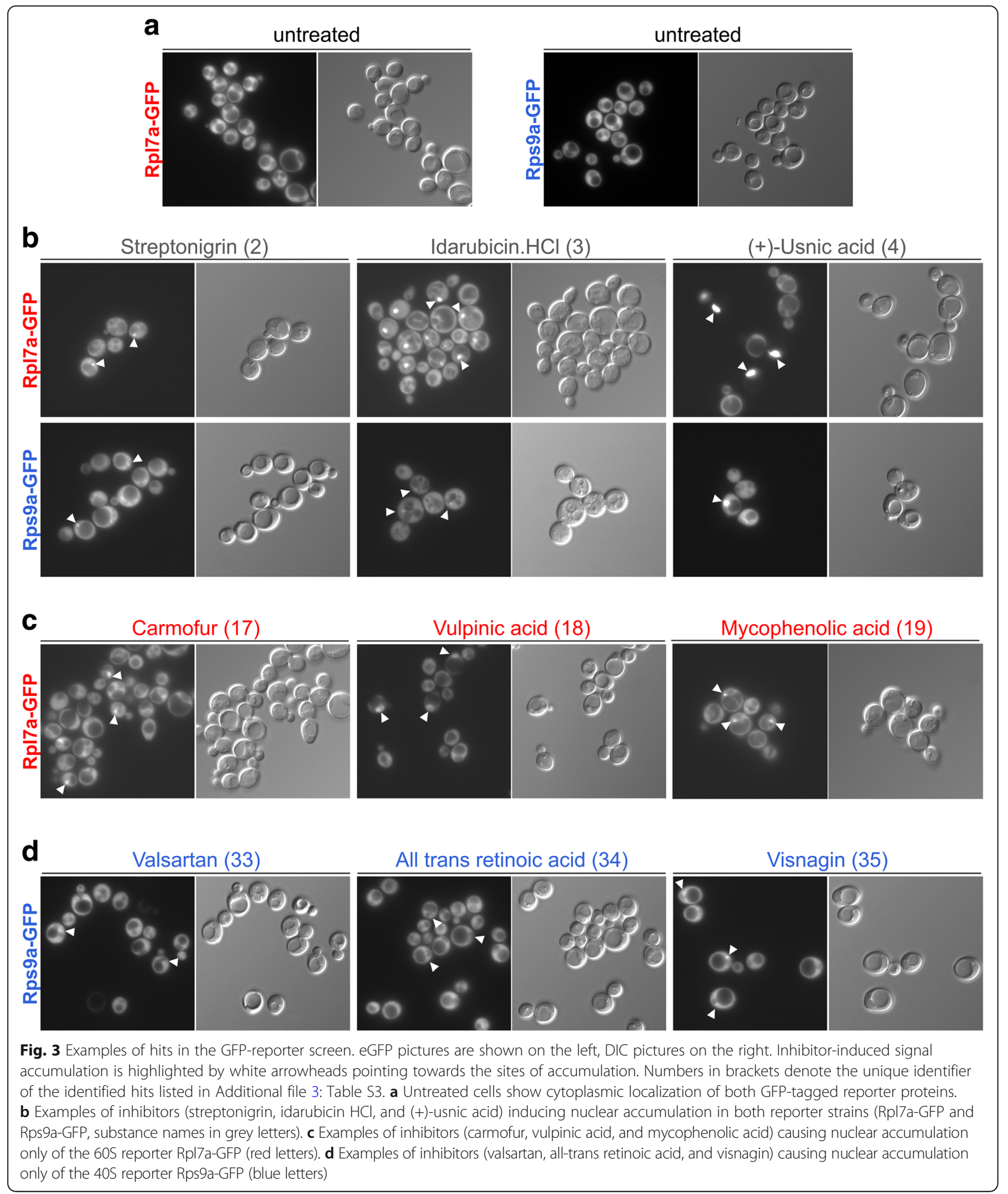

rRNAs or pre-rRNA spacer elements (Fig. 4a and Additional file 1: Figure S1). With this set of probes, we were able to monitor a variety of pre-rRNA precursors including (1) $35 \mathrm{~S}$ pre-rRNA, detectable by all probes; (2) $27 \mathrm{SA}_{2}$ pre-rRNA; (3) total $27 \mathrm{~S}$ pre-rRNA (including $27 \mathrm{SA}_{2}, 27 \mathrm{SA}_{3}$, and $27 \mathrm{SB}$ forms); (4) $7 \mathrm{~S}$ prerRNA; and (5) $20 \mathrm{~S}$ pre-rRNA. Additionally, two of the probes also detected the 23S RNA, which is generated by 
a

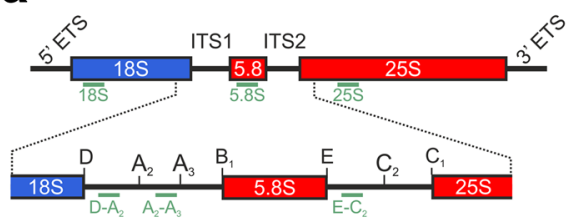

b

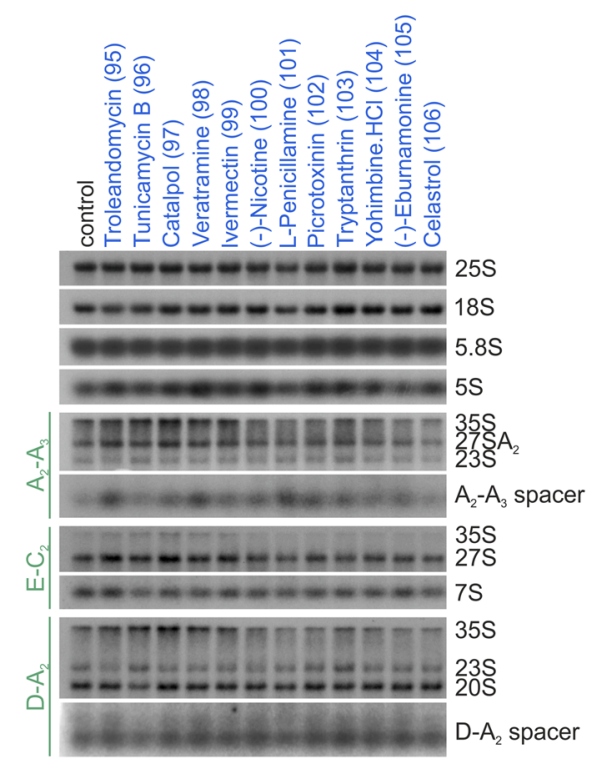

c

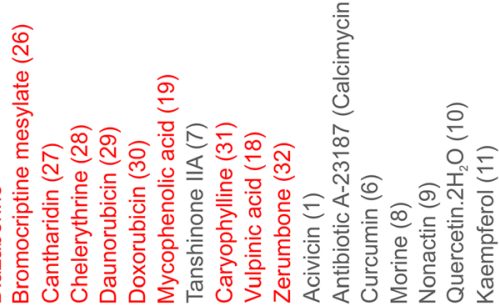

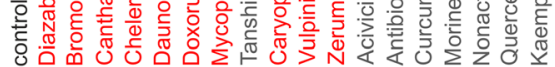

- -

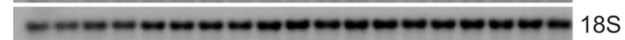

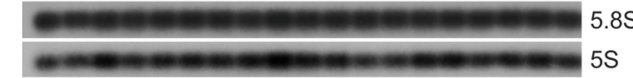

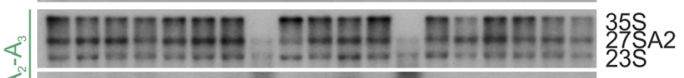

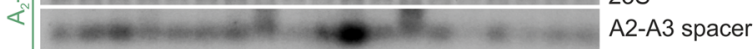

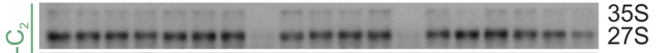

山े| - = -

|

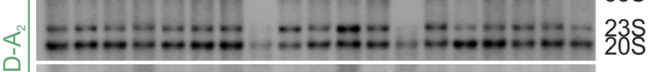

Len

d
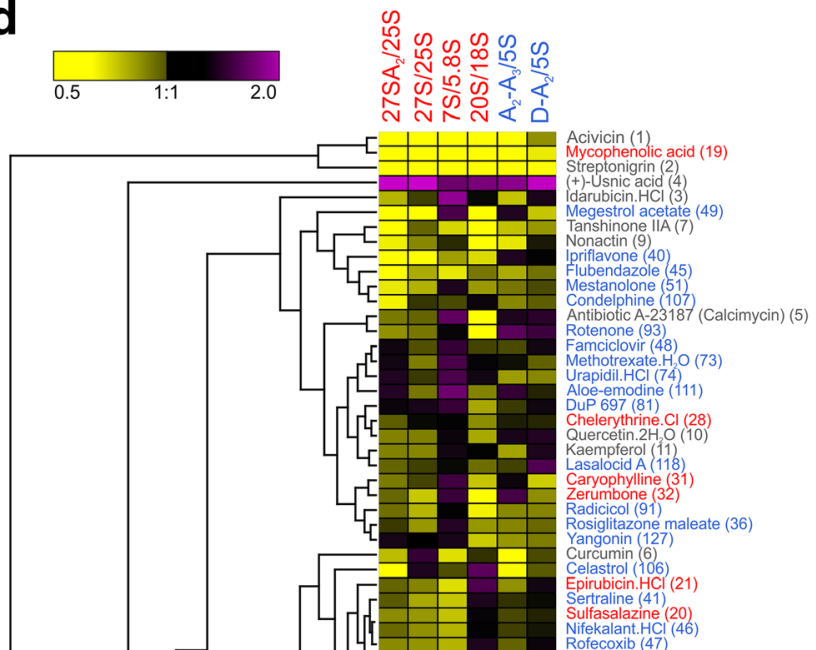

Rofecoxib (47). Na (42)
Pantoprazole. (Na)
Viramune (Nevirapine) (38)

Viramune (Nevirapine)
Itraconazole (22)

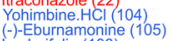

(sorhoifolin (108)

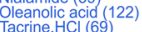

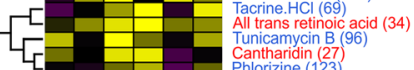

प्त

Phlorinine (12)
Carrotur (17)
Cefacolor ( 574$)$

Celaclor (54)

C.-Cotinine $77 C_{1}(79)$
Pramipexol.
Desoximetasone (55)

Desoximetassone $(55)$

Pergolicie mesylate (84)
Varigenin-7-O-glucoside (110)

Narigenin-7-O-glucoside (110)
Syringetine-3-glucoside (13)

Syringetine-3-glucoside
Trans-4-Cotininecarboxylic acid (112)

Troleandomycing
Veratramine
$(98)$

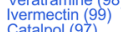

Catalpol (97)

$(-$-Nicotine 1000$)$
Methyytestosterone
(44)

Zileuton (52)

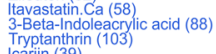

Fluticasone propionate (43)

Doxorubicin (30)

Ginkgolide $B(85)$
$\beta-E s t r a d i o l$
(80)

$\beta$-Estradiol (80)
Morine $(8)$

Cephadrine (119)
Rimcazole (57)

Parthenolide (89)

Barterini HCC (14)
bevocetirizine (63)

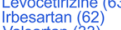

Valsartan (33)

Maprotiline. $\mathrm{HCl}(75)$

Pizotyline (78)

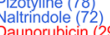

Daunorubicin 229
$( \pm)$ Kavain 187$)$

sicine (120) 1 (90)

Bromocriptine mesylate (26)

GR $89696(6)$
Donepezil $(60)$

etrahydropapaverine. $\mathrm{HCl}(116)$

Pancuronium (64)

Visnagin ( (35)

Crimebutine maleate (50)

Olanzapine (37) (8)

Parecoxib.Na (24)
Artemether 25 )

Picropodophlyllin (109)
Tetrayydrolipistatin (121)
Sinenseting

Rosmarinic acid (92)

Arbutin (128)

Gitoxigenin $(113)$
Harmane $(1414)$

Leucomisine (115)

Clotrimazole $(23)$
C-Oxocafestol (126)

-Oxocafestol
cisapride. $\mathrm{H}_{2} \mathrm{O}(67)$

Cinanserin $(65)$
Doxepin (68)

Bleomycin sulfate (16)

L-Penicillamine (101)

Fluphenazine.2 $\mathrm{HCl}$ (53)

Diazaborine (NII))

Fig. 4 (See legend on next page.) 
(See figure on previous page.)

Fig. 4 Inhibitors induce different rRNA processing defects. a Schematic picture of the longest rRNA precursor (35S pre-rRNA) containing the sequences of the mature $18 \mathrm{~S}$ rRNA, the $5.8 \mathrm{~S}$ rRNA and the $25 \mathrm{~S}$ rRNA, which are interrupted and flanked by internal (ITS) and external (ETS) transcribed spacers, respectively. The region encompassing ITS1 and ITS2 is enlarged and the main processing sites $\left(A_{2}, A_{3}, B_{1}, C_{1}, C_{2}, D\right.$, and E) are indicated. Hybridization sites of probes used in the northern blotting experiment are indicated by green bars. The entire processing pathway is displayed in Additional file 1: Figure S1. b, c Examples of northern blots after treatment with substances found in the $40 \mathrm{~S}$ reporter screen (b, blue lettering), in the 60S reporter screen (c, red lettering) or in both screens (c, grey lettering). The detected rRNA species are indicated on the right side, the probes used to detect the respective pre-rRNAs are indicated on the left side. The northern blots for all 128 compounds are shown in Additional file 1: Figures S10 and S11. d Hierarchical clustering of the indicated pre-rRNA/rRNA ratios. The color code in the heatmap indicates increased (purple) or decreased (yellow) levels of the respective precursors normalized to the mock control (DMSO) and then referenced to the respective mature rRNA in the same sample. Inhibitors found in the 605 reporter screen are marked by red lettering, inhibitors from the 405 screen are written in blue and inhibitors identified in both screens in grey. The control diazaborine was included once with the same DMSO concentration used in the screen with the $\mathrm{NIH}$ substances and once with the DMSO concentration used in the Enzo screen. Both conditions were found in the same cluster, demonstrating neglectable effects of the different DMSO concentrations

aberrant cleavage at site $\mathrm{A}_{3}$ upon a delay of the early $\mathrm{A}_{0}$, $A_{1}$, and $A_{2}$ processing steps (Additional file 1: Figure $S 1$ [114-116]). Additionally, two spacer fragments arising from endonucleolytic cleavages of $27 \mathrm{SA}_{2}$ and $20 \mathrm{~S}$ prerRNAs, the $\mathrm{A}_{2}-\mathrm{A}_{3}$ spacer and the D- $\mathrm{A}_{2}$ spacer, respectively, were detected in our analyses. As several substances caused alterations in the levels of these two spacer fragments, we also included them in our analyses.

Altogether, these experiments revealed a number of different pre-rRNA processing defects caused by the tested inhibitors. Examples of blots are shown in Fig. 4b (40S hits) and c (60S hits), while the northern blot analyses for all 128 substances are displayed in Additional file 1: Figures S10 and S11. Signals detected in the northern blots were densitometrically quantified and pre-rRNA signals were normalized to the mock control (DMSO). Due to the high stability of mature ribosomes, mature rRNA levels are expected to be largely unaltered after the short treatment period of $30 \mathrm{~min}$, allowing referencing of each precursor to the respective mature rRNA. Therefore, the calculated values represent ratios of pre-rRNA precursors relative to the respective mature rRNAs in each sample and are listed in Additional file 4: Table S4. Spacer fragments were referred to the $5 \mathrm{~S}$ rRNA due to the similar size. Subsequently, we performed hierarchical clustering of the signal ratios (Fig. 4d). 35S and $23 \mathrm{~S}$ pre-rRNAs were excluded from these analyses due to high variation in the quantifications caused by low levels in most samples and therefore low signal to noise ratios. The clustering analysis highlights that the tested inhibitors induced many different processing defects, leading either to accumulation or reduction of precursors. All substances causing accumulation or reduction of at least one pre-rRNA species by a factor of at least 1.5 are listed in Table 1 .

Although $35 \mathrm{~S}$ and $23 \mathrm{~S}$ signals were not considered for quantification and clustering analysis, substances leading to clear $35 \mathrm{~S}$ or $23 \mathrm{~S}$ accumulation were manually selected and are included in Table 1. For each of the different observed phenotypes, the respective inhibitor causing the strongest effect is additionally highlighted within the processing pathway in Fig. 5, emphasizing the good coverage of maturation steps targeted by the inhibitors.

\section{Inhibitors target a broad spectrum of maturation steps along the ribosome biogenesis pathway}

The wide range of different rRNA processing defects indicates that the set of novel inhibitors covers large parts of the ribosome biogenesis pathway ranging from the transcriptional level to very early nucleolar steps to late subunit-specific steps in the nucleoplasm. Three substances, acivicin, mycophenolic acid, and streptonigrin, caused a drastic reduction of all pre-rRNAs (Fig. 4, Table 1). Since all rRNA precursors are affected, we conclude that these substances cause a general block in rRNA transcription rather than targeting processing of specific precursors. In contrast to a general blockage of transcription, all other substances led to a reduction of few or only one rRNA precursor(s). This reduction of specific precursors may either be an indication for a blockage at an upstream maturation step or for instability of an intermediate, leading to its degradation. Tanshinone IIA, for instance, mainly led to a reduction of $27 \mathrm{SA}_{2}$ pre-rRNA; as however, also $20 \mathrm{~S}$ pre-rRNA levels were reduced, a maturation step upstream of the generation of these two precursors, for example $A_{2}$ cleavage or earlier steps, might be affected. Likewise, megestrol acetate and also most of the other substances mainly leading to $20 \mathrm{~S}$ reduction also showed reduced $27 \mathrm{~S}$ levels to some extent, suggesting that they might also exert their main effects before separation of the $40 \mathrm{~S}$ and $60 \mathrm{~S}$ maturation pathways. Interestingly, multiple inhibitors, including berberine $\mathrm{HCl}$, resulted in clear accumulation of the $23 \mathrm{~S}$ rRNA, indicative of delays in $\mathrm{A}_{0}, \mathrm{~A}_{1}$, and $\mathrm{A}_{2}$ processing. Only few of the tested substances caused significant $20 \mathrm{~S}$ pre-rRNA accumulation $((+)$-usnic acid, celastrol, epirubicin $\mathrm{HCl}$, narigenin-7-O-glucoside, isorhoifolin, parecoxib $\mathrm{Na}$, artemether, picropodophyllin, carmofur, trans-4-cotininecarboxylic acid). This is not surprising considering the facts that pre-40S particles 
Table 1 Inhibitors causing the strongest pre-rRNA processing phenotypes and the most affected intermediate (for a complete list of all changing pre-rRNAs, see Additional file 3: Table S3)

\begin{tabular}{|c|c|c|c|}
\hline \multirow{2}{*}{$\begin{array}{l}\text { Strongest pre-rRNA } \\
\text { phenotype }( \pm>1.5 x)\end{array}$} & \multicolumn{3}{|l|}{ Microscopy screen } \\
\hline & 605 hit & $40 S+605$ hit & $40 S$ hit \\
\hline All precursors gone & Mycophenolic acid (19) & Acivicin (1), streptonigrin (2) & \\
\hline $27 S A_{2} \downarrow^{*}$ & & Tanshinone IIA (7) & Flubendazole (45) \\
\hline $27 \mathrm{SA} \mathrm{A}_{2} \uparrow$ & Carmofur (17) & & $\begin{array}{l}\text { Valsartan (33), levocetirizine (63), } \\
\text { cinanserin (65), doxepin (68), cytarabine } \\
\text { (71), trans-4-cotininecarboxylic acid (112), } \\
\text { gitoxigenin (113) }\end{array}$ \\
\hline $275 \downarrow$ & & Acivicin (1) & Ipriflavone (40) \\
\hline $275 \uparrow$ & & (+)-Usnic acid (4), syringetine-3-glucoside (13) & \\
\hline $7 S \downarrow$ & & & Tunicamycin B (96), catalpol (97) \\
\hline $7 \mathrm{~S} \uparrow$ & & $\begin{array}{l}\text { Idarubicin } \mathrm{HCl}(3) \text {, cefaclor (54), } \\
\text { desoximetasone (55) }\end{array}$ & $\begin{array}{l}\text { Fluphenazine } 2 \mathrm{HCl}(53)^{* *} \text {, pergolide } \\
\text { mesylate (84) }\end{array}$ \\
\hline $20 S \downarrow$ & & Antibiotic A-23187 (calcimycin) (5), nonactin (9) & $\begin{array}{l}\text { All trans retinoic acid (34), megestrol } \\
\text { acetate (49), rotenone (93) }\end{array}$ \\
\hline $\mathrm{A}_{2}-\mathrm{A}_{3} \mathrm{sp} . \downarrow$ & & Curcumin (6) & \\
\hline $\mathrm{A}_{2}-\mathrm{A}_{3} \mathrm{sp} . \uparrow$ & Vulpinic acid (18) & & $\begin{array}{l}\text { Fluphenazine } 2 \mathrm{HCl}(53)^{* *} \text {, rimcazole }(57) \text {, } \\
\text { thapsigargin (94), troleandomycin (95), } \\
\text { veratramine (98), (-)-nicotine (100), } \\
\text { L-penicillamine (101), picrotoxinin (102), }\end{array}$ \\
\hline$D-A_{2} \uparrow$ & & & Icariin (39) \\
\hline \multicolumn{4}{|c|}{ Additional strong pre-rRNA changes (manually curated) } \\
\hline $35 S \uparrow$ & $\begin{array}{l}\text { Parecoxib sodium (24), } \\
\text { zerumbone (32) }\end{array}$ & $\begin{array}{l}\text { Tanshinone IIA (7), morine (8), nonactin (9), } \\
\text { senecionine (15), bleomycin sulfate (16) }\end{array}$ & $\begin{array}{l}\text { Visnagin (35), zileuton (52), } \\
\text { hexamethylenebisacetamide } \\
\text { (66), indatraline } \mathrm{HCl}(70), \text { cytarabine } \\
(71), \text { naltrindole }(72) \text {, uradipil } \mathrm{HCl}(74), \\
\text { DuP } 697 \text { (81), vindesine sulfate (82), } \\
\text { clobenpropit (83), pergolide mesylate } \\
\text { (84), catalpol (97), veratramine (98), } \\
\text { ivermectin (99), (-)-nicotine (100), } \\
\text { tryptanthrin (103), celastrol (106), } \\
\text { isorhoifolin (108), narigenin-7-O- } \\
\text { glucoside (110), leucomisine (115), } \\
\text { tetrahydropapaverine HCl (116), } \\
\text { tetrahydrolipistatin (121), phlorizine } \\
\text { (123), diosmin (124), }\end{array}$ \\
\hline Aberrant $23 S \uparrow$ & Carmofur (17), vulpinic acid (18) & Tanshinone IIA (7), berberine $\mathrm{HCl}(14)$ & Yangonin (127) \\
\hline
\end{tabular}

* $\uparrow$ denotes accumulation, $\downarrow$ denotes reduction of the respective precursor

** Listed twice due to equally strong effects

(Substance identifier no., compare Additional file 3: Table S3)

contain only few assembly factors and moreover are exported rapidly after $\mathrm{A}_{2}$ cleavage, both minimizing the pool of potential targets to be identified by nuclear accumulation of a $40 \mathrm{~S}$ subunit reporter.

In addition to substances interfering with very early processing events, several substances clearly affected later, $60 \mathrm{~S}$ subunit-specific stages, deducible from their characteristic rRNA processing defect. Several of these compounds caused $27 \mathrm{SA}_{2}$ accumulation, indicative of an early $60 \mathrm{~S}$-specific processing defect, with the 5-FU derivative carmofur showing the strongest phenotype. Notably, we found that yeast cells, in which genes encoding components of the exosome (i.e., Rrp6, Rrp43 and Mtr4), a multi-subunit complex involved in 7S pre-rRNA processing, had been deleted, were hypersensitive to carmofur (Additional file 1: Figure S12). Moreover, previous experiments demonstrated that deletion of exosome components caused hypersensitivity to 5 -FU $[117,118]$. Together, these results strengthen the hypothesis that carmofur and 5-FU directly target the ribosome biogenesis pathway.

Another substance, syringetine-3-glucoside, showed the strongest accumulation of total $27 \mathrm{~S}$ signal, although also $27 \mathrm{SA}_{2}$ accumulated, suggesting a delay in maturation of $27 \mathrm{SA}_{2}$ as well as $27 \mathrm{SB}$ precursors. Alternatively, the inhibitor-induced 27SB accumulation might cause a secondary block in processing of the earlier $27 \mathrm{SA}_{2}$. 


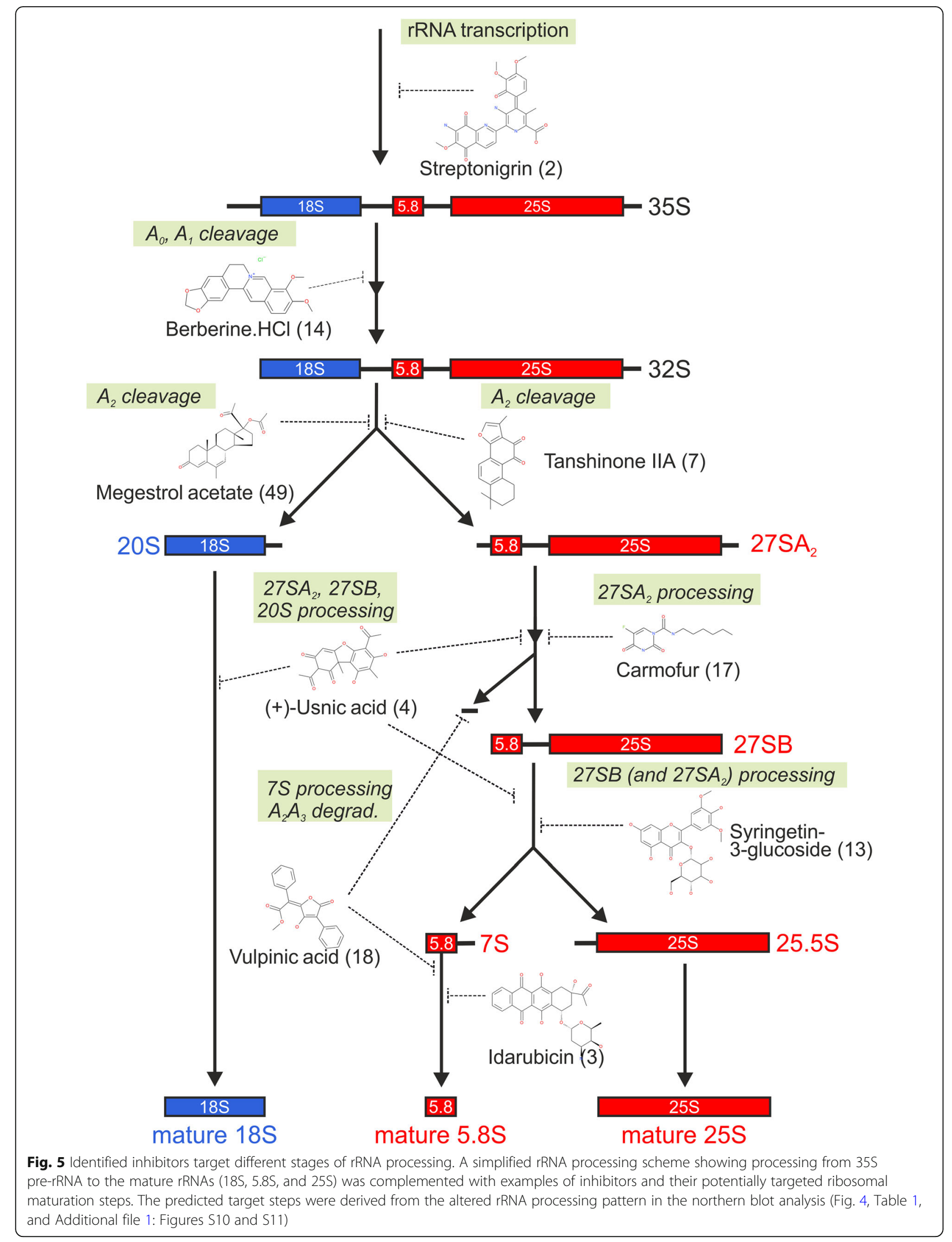


Even later maturation steps were affected by vulpinic acid. This lichen secondary product led to accumulation of 7S pre-rRNA, but also of the aberrant 23S RNA, as well as to an accumulation of the small $\mathrm{A}_{2}-\mathrm{A}_{3}$ spacer fragment. A similar albeit weaker effect (accumulation of $7 \mathrm{~S}, 23 \mathrm{~S}$, and $\mathrm{A}_{2}-\mathrm{A}_{3}$ spacer) was also observed with fluphenazine $2 \mathrm{HCl}$. This slowdown in earlier processing steps $\left(\mathrm{A}_{0}, \mathrm{~A}_{1}\right.$, and $\left.\mathrm{A}_{2}\right)$ upon $7 \mathrm{~S}$ accumulation is another demonstration that inhibition of late steps can rebound on earlier events [29].

A late pre-60S maturation defect was also observed for idarubicin, which caused the accumulation of $7 \mathrm{~S}$ pre-rRNA, suggesting it inhibits nucleoplasmic 60S maturation steps. Idarubicin belongs to the compound group of rubicins, of which three additional members (daunorubicin, doxorubicin, and epirubicin) showed up in our screen. Since doxorubicin was reported previously to block rRNA transcription in human cells $[24,119]$ we tested two rubicins for effects on pre-ribosome maturation in mammalian cell culture. Indeed, doxorubicin and epirubicin lead to changed nucleolar morphology and nucleoplasmic accumulation of an Rpl27-GFP reporter construct in HeLa cells (Additional file 1: Figure S13).

In summary, the wide range of rRNA processing defects observed upon inhibitor treatment suggests that large parts of the ribosome maturation pathway can be targeted by low molecular weight inhibitors.

\section{Discussion}

In this study, we performed a microscopy-based screen to identify a large set of novel inhibitors of the ribosome biogenesis pathway. In total, 128 substances caused accumulation of pre-ribosomal particles in the nucleus. Three of these substances interfered with rRNA transcription: streptonigrin, acivicin, and mycophenolic acid led to an almost complete disappearance of pre-rRNAs after $30 \mathrm{~min}$ of treatment (Fig. 4 and Additional file 1: Figure S11). Intriguingly, the accumulation of the reporter constructs in the absence of any detectable prerRNA may be an indication for a nucleolar deposition/ retention system for ribosomal proteins. Acivicin and mycophenolic acid are known to inhibit purine and/or pyrimidine synthesis. Their effect on rRNA transcription can be explained by the fact that rRNA synthesis is presumably by far the biggest nucleotide consumer in growing cells. Consistent with this suggestion, the effects of these compounds on transcription are not specific for RNA polymerase I, since the NRD1 mRNA in comparison to the long-lived ACT1 mRNA showed a decrease after $30 \mathrm{~min}$ of treatment (Additional file 1: Figure S11). This indicates that also transcription by RNA polymerase II is affected. Streptonigrin acts differently and is known to complex with DNA and thereby affects not only transcription, but also replication [120].
Our main interest was however the identification of inhibitors targeting maturation steps downstream of transcription. Indeed, all other substances causing phenotypes in the northern blot analysis (Fig. 4 and Table 1) inhibited rRNA maturation and not transcription. This analysis shows that our inhibitors cover many different maturation steps along the ribosome biogenesis pathway.

It is noteworthy that many substances caused very early defects, obvious from accumulation of the GFP reporters, and in particular Rps9a-GFP, in small dots in the nucleolus (Additional file 1: Figure S9). The most likely explanation is that the majority of known ribosome assembly factors and therefore also the highest number of potential targets for inhibition participate in very early, nucleolar maturation steps. This suggests that many of the identified substances interfere with steps of ribosome biogenesis preceding $\mathrm{A}_{2}$ cleavage and/or incorporation of Rpl7. This would explain why only 33 compounds were identified to cause nuclear accumulation of the large subunit reporter Rpl7a-GFP, while 110 substances scored positive in the screen using the small subunit reporter Rps9a-GFP.

The validity of our results is confirmed by inhibitors identified in our screen which have already been linked to ribosome biogenesis. One prime example is the pyrimidine analogue carmofur, a derivative of 5-fluorouracil containing an additional carbamoyl moiety that allows oral administration of the drug [62, 117, 118]. This modification makes the spectrum of targets even broader since carmofur was shown to be also effective in 5-FU-resistant cells [121-124]. Both 5-FU and carmofur are widely used as chemotherapeutic agents despite the fact that their manifold effects on the cell are not fully understood. 5-FU is incorporated into RNA and interferes with multiple nucleotide-related pathways including rRNA transcription and processing [117, 125-128]. Based on their various effects on RNA metabolism, it was anticipated that these pyrimidine analogues might also affect the processing of pre-rRNAs [24, 117, 129]. Indeed, several studies suggested a link between the action of pyrimidine analogues and components of the exosome, which catalyzes the $3^{\prime}-5^{\prime}$ trimming of the $7 \mathrm{~S}$ pre-rRNA [117, 118, 126, 127, 130]. In line with these suggestions, we observed super-sensitivity of exosome mutants also to carmofur and detected increased levels of $7 \mathrm{~S}$ pre-rRNA in our study, even though the most prominent effect of the drug was an accumulation of $27 \mathrm{SA}_{2}$ pre-rRNA (Fig. 4d). Notably, also the known exosome target NRD1 mRNA [131] accumulated after carmofur treatment, further supporting a direct action of the compound on the exosome (Additional file 1: Figure S10). Interestingly, cantharidin also caused a strong accumulation of the NRD1 mRNA. Treatment of mammalian cells with cantharidin was recently shown to 
result in overexpression of several components of the $3^{\prime}-5^{\prime}$ decay pathway, including two core components of the exosome [132], which may be an indication for a connection of the drug target to the $3^{\prime}-5^{\prime}$ RNA decay machinery.

Another interesting example for a substance group whose members scored as hits in our screen is the rubicins. These compounds are thought to block DNA replication by intercalating into the DNA or inhibiting topoisomerase II [133-136] and are widely used for clinical treatment of solid tumors. In addition to the reported effect of doxorubicin on pre-rRNA transcription in yeast and human fibrosarcoma cells $[24,119]$, we found that rubicins interfere with pre-ribosome maturation and export in HeLa cells. In our screen, the individual rubicins caused different patterns of pre-rRNA processing defects in yeast and hence seem to affect different maturation steps in ribosome biogenesis. However, it has to be noted that these differences may also be the result of different susceptibility of yeast to the individual rubicins. While a unified concentration and treatment period was necessary for this large-scale study, the MICs, as well as the optimal concentrations and treatment periods with the inhibitors, will have to be determined in detail in future studies.

Another compound previously linked to ribosome biogenesis is the lichen secondary metabolite vulpinic acid. Haplo-insufficiency profiling experiments previously showed that among other strains, the heterozygous deletion of the pre-60S assembly factor YTM1 resulted in increased vulpinic acid sensitivity [137]. This result further supports that vulpinic acid targets ribosome biogenesis and suggests that the substance acts in close functional proximity to Ytm1. In our experiment, vulpinic acid treatment led to accumulation of the 7S and $23 \mathrm{~S}$ pre-rRNAs, as well as a striking accumulation of the $\mathrm{A}_{2}-\mathrm{A}_{3}$ spacer fragment known to be degraded mainly by the $5^{\prime}-3^{\prime}$ exonuclease Rat1 [138]. However, since the level of the $\mathrm{A}_{0}-\mathrm{A}_{1}$ spacer, which is also a target of Rat1, was unaffected by Vulpinic acid (data not shown), a direct inhibition of Rat1 is unlikely. Moreover, conditional ytm1 mutants, YTM1 depletion, or over-expression of a dominant negative YTM1 allele showed different prerRNA processing patterns and mainly caused 27SB pre-rRNA accumulation $[139,140]$. Therefore it is also unlikely that Ytm1 is the direct target of the inhibitor. Further studies will be necessary to reveal the precise target of vulpinic acid in ribosome biogenesis.

All these examples of compounds that were reported independently from our study to affect ribosome biogenesis validate the results of our screen. However, the vast majority of the small molecules identified in this screen were for the first time recognized as inhibitors of the ribosome biogenesis pathway. This demonstrates that our screening method can successfully be used to mine a complex pathway such as ribosome formation for potent and specific inhibitors. Since the identified inhibitors cause a broad range of pre-rRNA processing changes, they cover many different steps of the ribosomal maturation cascade. Therefore our study provides a comprehensive toolbox of novel inhibitors which allows to investigate the highly dynamic process of ribosome synthesis.

Ribosome biogenesis is tightly interwoven with numerous other pathways. This is supported by our observation that several inhibitors of other cellular processes also scored positive in our screen for ribosome biogenesis inhibitors, potentially revealing up to now unrecognized regulatory cross-talks. Tunicamycin B, for example, activates the unfolded protein response pathway and was additionally shown to downregulate transcription of ribosomal protein genes [141-143]. Similarly, methotrexate blocks dihydrofolate reductase [144], thereby affecting nucleotide synthesis, transcription of rRNA [24], and likely also formation of $S$-adenosylmethionine $[145,146]$ which is required for methylation of rRNA.

Although the identification of the precise targets of the here identified ribosome biogenesis inhibitors remains a task for future studies, selective inhibitors will become valuable tools to facilitate the exploration of hitherto not well-understood steps of the pathway. The high number of potential drug targets will also open up novel avenues for chemotherapy.

\section{Conclusions}

The results from our screen provide for the first time a broad set of inhibitors targeting various steps of ribosome biogenesis. These compounds will not only prove valuable to investigate this highly interesting pathway but also to identify novel drug targets. Remarkably, many of the identified substances were previously shown to interfere with growth of tumor cells, are currently being investigated in clinical trials, or have already been used for clinical cancer treatment [24, 26] (see also Additional file 3: Table S3). This finding underlines the crucial importance of ribosome biogenesis for fast proliferating cells including tumor cells and eukaryotic pathogens. This suggests that targeting ribosome biogenesis might be an efficient strategy for treatment of infectious diseases or malignant tumors.

\section{Methods}

\section{Yeast strains}

In order to prevent interference of the Ade-pigment in the W303 ade2 strain with fluorescence microscopy, wild-type $A D E 2$ was integrated into the strain by homologous recombination, resulting in the white W303 derivative C303. This strain was also used for northern blot analyses. To generate the small and the large 
subunit export reporter strains, the chromosomal copies of $R P L 7 a$ and $R P S 9 a$ were C-terminally fused to GFP by homologous recombination using a HIS3MX6 selection marker. The integration cassettes were generated by PCR using the pFA6a-HIS3MX6 plasmid [147] as template and $55 \mathrm{bp}$ of gene-specific sequences as primer overhangs. For co-localization experiments, selected nuclear compartment markers (Nic96, Nop58, Hho1) were $\mathrm{C}$-terminally fused to $3 \mathrm{x}$-mCherry in the GFP-reporter strains, also done by homologous recombination. Plasmid pFA6a-3mcherry-hphNT1 served as template for the generation of the recombination cassette. The genotypes of all strains are listed in Additional file 5: Table S5 [148].

\section{Compound libraries}

The NIH clinical collection, provided by the National Institute of Health (NIH), USA, included 446 clinically relevant compounds in a concentration of $10 \mathrm{mM}$ dissolved in DMSO. The Screen-Well Natural Product Library Version 7.4 from Enzo Life Sciences included 502 individual purified compounds in DMSO with a final concentration of $2 \mathrm{mg} / \mathrm{ml}$. Full lists of all tested inhibitors are provided in Additional file 2: Tables S1 and S2.

\section{Microscopy-based screening}

Strains C303a Rpl7a-GFP and C303a Rps9a-GFP were grown in 96-well deep-well plates in synthetic dextrose medium lacking histidine (SD-his) at $28^{\circ} \mathrm{C}$ to an $\mathrm{OD}_{600}$ of 0.4 (early log-phase). Subsequently, inhibitors were added at a final concentration of $50 \mu \mathrm{M}$ and cells were further incubated for at least $3 \mathrm{~h}$. Diazaborine was used as a control with a final concentration of $18 \mu \mathrm{M}$. DMSO alone, the solvent of the tested substances, caused no nuclear accumulation of the ribosomal reporter proteins in tested concentrations up to $6 \%$. Consequently, untreated cells without DMSO served as negative control. Subsequently, fluorescence microscopy was performed using a Zeiss Axioskop Microscope with a narrow band enhanced GFP filter from Zeiss. At least four representative pictures were recorded for each substance, and pictures were independently evaluated by two researchers for nucle(ol)ar accumulation of the reporter proteins. All substances were screened twice, and compounds leading to nuclear accumulation of a reporter protein were confirmed in a third round of analysis.

For co-localization microscopy experiments, inhibitortreated cells of strains additionally expressing $3 \mathrm{x}$-mCherry tagged nuclear compartment marker proteins were investigated using a Leica DM6 B Microscope equipped with a $\times 100 / 1.4$ Plan APO objective and narrow band GFP or RHOD ET filters. For imaging, the high-resolution DFC9000GT camera and the LASX premium software were used.

\section{RNA isolation and northern blotting}

C303a cells were grown in SD medium at $30{ }^{\circ} \mathrm{C}$ to an $\mathrm{OD}_{600}$ of $\sim 0.7$ (log-phase). The inhibitors were added at a final concentration of $50 \mu \mathrm{M}$ to $2 \mathrm{ml}$ of culture each. Addition of the NIH inhibitors led to a final DMSO concentration of $0.5 \%$ in the culture. The Enzo inhibitors, provided by the company in $2 \mathrm{mg} / \mathrm{ml}$ concentration, were adjusted with DMSO to $50 \mu \mathrm{M}$ resulting in final DMSO concentrations of $\sim 2 \%$ in the culture. Separate negative controls were grown for the NIH and Enzo substances with $0.5 \%$ and $2 \%$ DMSO respectively. Additionally, diazaborine was used as a positive control for screens with both libraries and was added with the corresponding amounts of DMSO.

After inhibitor treatment for $30 \mathrm{~min}$, cells were harvested and suspended in $200 \mu \mathrm{l}$ lysis buffer $(10 \mathrm{mM}$ Tris- $\mathrm{HCl} \mathrm{pH}$ 7.5, $10 \mathrm{mM}$ EDTA, 0.5\% SDS). After addition of $200 \mu \mathrm{l}$ glass beads $(0.5 \mathrm{~mm}$ diameter), cells were mechanically disrupted by vigorous shaking for $3 \mathrm{~min}$. RNA was extracted from the lysates by phenol-chloroform-isoamylalcohol (25:24:1; three times), followed by chloroform-isoamylalcohol (24:1) extraction and ethanol precipitation. Three micrograms of RNA per sample was separated on 1.5\% MOPS-agarose gels. The RNA was transferred overnight onto a Hybond-N nylon membrane (Amersham Biosciences) and then UV cross-linked to the membrane. Except for the E/C2, anti-ACT1, and anti-NRD1 probes $\left(37^{\circ} \mathrm{C}\right)$, hybridization was performed overnight at $42^{\circ} \mathrm{C}$ in $500 \mathrm{mM} \mathrm{NaPO}_{4}$ buffer, pH 7.2, 7\% SDS, $1 \mathrm{mM}$ EDTA using $5{ }^{\prime}-{ }^{32}$ P-labeled oligonucleotide probes with the following sequences: $18 \mathrm{~S}$ rRNA: CATGGCTTAATCTTTGAGAC, 25S rRNA: CTC CGCTTATTGATATGC, 5.8S rRNA, GCGTTCTTCATCGATGC, 5S rRNA: GGTCACCCACTACACTACTCGG, A2-A3: TGTTACCTCTGGGCCC, E-C2: GGCCAGCAA TTTCAAGTTA, D-A2: GACTCTCCATCTCTTGTCTT CTTG, anti-ACT1: CCGGCAGATTCCAAACCCAAAA CAGAAGGATGGA, anti-NRD1: GCTCATCGGGGTAT AAGTGGTGATTGTTTGTGC [131]. The membranes were washed three times for $20 \mathrm{~min}$ at $42^{\circ} \mathrm{C}$ in $40 \mathrm{mM}$ $\mathrm{NaPO}_{4}$ buffer, pH 7.2, $1 \%$ SDS. Membranes were regenerated by washing in $1 \%$ SDS. Each sample was analyzed two times with independent gels and hybridizations.

Signals were detected by autoradiography and quantified using the ImageLab 5.2 software (Biorad). Quantified signals were normalized using the signals of the mock control (DMSO), which was loaded at least once per 20 treated samples. Ratios of precursors to mature rRNAs were calculated. $27 \mathrm{~S}$ pre-rRNAs were referenced to mature $25 \mathrm{~S}$ rRNA levels, $20 \mathrm{~S}$ pre-rRNA to mature $18 \mathrm{~S}$ rRNA, and 7S pre-rRNA to mature $5.8 \mathrm{~S}$ rRNA. The spacer fragments were referenced to the $5 \mathrm{~S}$ pre-rRNA due to the similar size. Means of the two ratios were calculated from the two northern blot rounds (values from the two individual rounds and mean values are listed in 
Additional file 4: Table S4). The mean values were then transformed into logarithmic values (basis 2) and loaded into the Genesis software provided by the Institute for Genomics and Bioinformatics, Graz University of Technology [149]. The data were subjected to hierarchical clustering using the average linkage agglomeration rule.

\section{Fluorescence microscopy of HeLa ${ }^{\text {Rpl7-GFP }}$ cells}

HeLaRpl27-GFP cells [150] stably expressing the ribosomal reporter protein Rpl27-GFP were cultured in Gibco FluoroBrite $^{\text {тм }}$ DMEM medium supplemented with 10\% Fetal bovine serum and GlutaMax (all Thermo Scientific) for $24 \mathrm{~h}$ before treatment with $1 \mu \mathrm{M}$ of the indicated compounds for $5 \mathrm{~h}$ and inspection using a Leica SP5 confocal microscope and a HCX PL APO $\times 25$ objective.

\section{Additional files}

\section{Additional file 1: Figure S1. Yeast rRNA processing pathway.}

Figure S2. Inhibitors causing nuclear accumulation of both the Rpl7a-GFP (60S) and the Rps9a-GFP (40S) reporter. Related to Fig. 3. Figure S3. Inhibitors causing nuclear accumulation of the Rpl7a-GFP (60S) reporter. Related to Fig. 3. Figure S4-S8. Inhibitors causing nuclear accumulation of the Rps9a-GFP (40S) reporter. Related to Fig. 3. Figure S9. Different classes of localization phenotypes upon inhibitor treatment. Figure S10. rRNA processing phenotypes caused by the inhibitors from the NIH inhibitor collection. Related to Fig. 4. Figure S11. rRNA processing phenotypes caused by the inhibitors from the Enzo inhibitor collection. Related to Fig. 4. Figure S12. Deletion of exosome factors cause hypersensitivity to Carmofur. Figure S13. Treatment with doxorubicin and epirubicin causes nucleoplasmic accumulation of an Rpl27-GFP reporter and nucleolar fragmentation in HeLa cells. (DOCX 20739 kb)

Additional file 2: Table S1. Complete list of compounds contained in the "NIH clinical collection" as provided by the distributor. Table S2. Complete list of compounds contained in the "Enzo Natural Product Library" as provided by the distributor. (PDF $1086 \mathrm{~kb}$ )

Additional file 3: Table S3. All hits of the microscopy screen including a complete list of all changing pre-rRNAs $( \pm>1.5 \mathrm{x}$ ) for substances listed in Table 1 and documented references to activities against cancer cells. (PDF $78 \mathrm{~kb}$ )

Additional file 4: Table S4. pre-rRNA precursor alterations after inhibitor treatment. Calculated ratios (pre-rRNA/mature rRNA) from quantifications of two northern blot experiments (round 1 and round 2 plus mean). The blots corresponding to round 1 are shown in Additional file 1: Figures S10 and S11. (XLSX $56 \mathrm{~kb}$ )

Additional file 5: Table S5. Saccharomyces cerevisiae strains used in this study. (PDF 244 kb)

\section{Acknowledgements}

The expert help of Mirjam Pennauer in early stages of this work is kindly acknowledged.

\section{Funding}

Austrian Science Fund FWF [P26136, P29451 to H.B.; P 28874, P 27996 to $\mathrm{BP}]$. The early phase of the project was supported by the unconventional research initiative of the rectorate of the Karl-Franzens University Graz initiated by Vice Rector Prof. Dr. Peter Scherrer.

\section{Availability of data and materials}

All data generated or analyzed during this study are included in this published article [and its supplementary information files (Additional files 1, 2, 3, 4 and 5)].

\section{Authors' contributions}

$D A, B P$, and HB conceived the study. DA, BP, IR, LK, MPrattes, GZ, MPertl, ML, $H W$, and $H B$ performed the experiments and analyzed the data. DA, BP, MPrattes, LK, and HB wrote the manuscript. All authors critically read and revised the manuscript. All authors read and approved the final manuscript.

Ethics approval and consent to participate

Not applicable.

\section{Consent for publication}

Not applicable.

Competing interests

The authors declare that they have no competing interests.

\section{Publisher's Note}

Springer Nature remains neutral with regard to jurisdictional claims in published maps and institutional affiliations.

\section{Author details}

${ }^{1}$ Institute of Molecular Biosciences, University of Graz, Humboldtstrasse 50/ EG, A-8010 Graz, Austria. ${ }^{2}$ Present address: Department of Cancer Systems Imaging, The University of Texas MD Anderson Cancer Center, Houston, TX, USA.

Received: 25 February 2019 Accepted: 14 May 2019

Published online: 10 June 2019

References

1. Baßler J, Hurt E. Eukaryotic ribosome assembly. Annu Rev Biochem. 2019;88: 8.1-8.26:26.

2. Kressler D, Hurt E, Baßler J. A puzzle of life: crafting ribosomal subunits. Trends Biochem Sci. 2017;42(8):640-54

3. Peña C, Hurt E, Panse VG. Eukaryotic ribosome assembly, transport and quality control. Nat Struct Mol Biol. 2017;24(9):689-99.

4. Woolford JL, Baserga SJ. Ribosome biogenesis in the yeast Saccharomyces cerevisiae. Genetics. 2013:195(3):643-81.

5. Dragon F, Gallagher JEG, Compagnone-Post PA, Mitchell BM, Porwancher KA, Wehner KA, et al. A large nucleolar U3 ribonucleoprotein required for 185 ribosomal RNA biogenesis. Nature. 2002;417(6892):967-70.

6. Grandi P, Rybin V, Baßler J, Petfalski E, Strauß D, Marzioch M, et al. 90 S preribosomes include the $35 \mathrm{~S}$ pre-rRNA, the U3 snoRNP, and 40 S subunit processing factors but predominantly lack 605 synthesis factors. Mol Cell. 2002:10(1):105-15.

7. Barandun J, Hunziker M, Klinge S. Assembly and structure of the SSU processome-a nucleolar precursor of the small ribosomal subunit. Curr Opin Struct Biol. 2018:49:85-93.

8. Cerezo E, Plisson-Chastang C, Henras AK, Lebaron S, Gleizes P-E, O'Donohue $M-F$, et al. Maturation of pre-40S particles in yeast and humans. Wiley Interdiscip Rev RNA. 2019;10(1):e1516.

9. Konikkat $\mathrm{S}$, Woolford JL. Principles of 605 ribosomal subunit assembly emerging from recent studies in yeast. Biochem J. 2017:474(2):195-214.

10. Henras AK, Plisson-Chastang C, O'Donohue M-F, Chakraborty A, Gleizes P-E. An overview of pre-ribosomal RNA processing in eukaryotes. Wiley Interdiscip Rev RNA. 2015;6(2):225-42.

11. Fernández-Pevida A, Kressler D, de la Cruz J. Processing of preribosomal RNA in Saccharomyces cerevisiae. Wiley Interdiscip Rev RNA. 2015;6(2):191-209.

12. Bradatsch B, Leidig C, Granneman S, Gnädig M, Tollervey D, Böttcher B, et al. Structure of the pre-60S ribosomal subunit with nuclear export factor Arx 1 bound at the exit tunnel. Nat Struct Mol Biol. 2012;19(12):1234-41.

13. Yao W, Roser D, Köhler A, Bradatsch B, Baßler J, Hurt E. Nuclear export of ribosomal 60S subunits by the general mRNA export receptor Mex67-Mtr2. Mol Cell. 2007;26(1):51-62.

14. Ameismeier M, Cheng J, Berninghausen O, Beckmann R. Visualizing late states of human 40S ribosomal subunit maturation. Nature. 2018;558(7709):249.

15. Barrio-Garcia C, Thoms M, Flemming D, Kater L, Berninghausen O, Baßler J, et al. Architecture of the Rix1-Rea1 checkpoint machinery during pre-60Sribosome remodeling. Nat Struct Mol Biol. 2016;23(1):37-44.

16. Cheng J, Kellner N, Berninghausen O, Hurt E, Beckmann R. 3.2-Å-resolution structure of the 905 preribosome before A1 pre-rRNA cleavage. Nat Struct Mol Biol. 2017;24(11):954-64. 
17. Kater L, Thoms M, Barrio-Garcia C, Cheng J, Ismail S, Ahmed YL, et al. Visualizing the assembly pathway of nucleolar pre-60S ribosomes. Cell. 2017; 171(7):1599-1610.e14.

18. Kornprobst M, Turk M, Kellner N, Cheng J, Flemming D, Koš-Braun I, et al. Architecture of the $90 \mathrm{~S}$ pre-ribosome: a structural view on the birth of the eukaryotic ribosome. Cell. 2016;166(2):380-93.

19. Sanghai ZA, Miller L, Molloy KR, Barandun J, Hunziker M, Chaker-Margot M, et al. Modular assembly of the nucleolar pre-60S ribosomal subunit. Nature. 2018;556(7699):126-9.

20. Sun Q, Zhu X, Qi J, An W, Lan P, Tan D, et al. Molecular architecture of the $90 S$ small subunit pre-ribosome. Elife. 2017;6:e22086.

21. Zhou D, Zhu X, Zheng S, Tan D, Dong M-Q, Ye K. Cryo-EM structure of an early precursor of large ribosomal subunit reveals a half-assembled intermediate. Protein Cell. 2019;10(2):120-30.

22. Warner JR. The economics of ribosome biosynthesis in yeast. Trends Biochem Sci. 1999;24(11):437-40.

23. Brighenti $E$, Treré $D$, Derenzini $M$. Targeted cancer therapy with ribosome biogenesis inhibitors: a real possibility? Oncotarget. 2015;6(36): 38617-27.

24. Burger K, Mühl B, Harasim T, Rohrmoser M, Malamoussi A, Orban M, et al. Chemotherapeutic drugs inhibit ribosome biogenesis at various levels. J Biol Chem. 2010;285(16):12416-25.

25. Catez F, Dalla Venezia N, Marcel V, Zorbas C, Lafontaine DLJ, Diaz J-J. Ribosome biogenesis: an emerging druggable pathway for cancer therapeutics. Biochem Pharmacol. 2019;159:74-81.

26. Derenzini M, Montanaro L, Trerè $D$. Ribosome biogenesis and cancer. Acta Histochem. 2017;119(3):190-7.

27. Loibl M, Klein I, Prattes M, Schmidt C, Kappel L, Zisser G, et al. The drug diazaborine blocks ribosome biogenesis by inhibiting the AAA-ATPase Drg1. J Biol Chem. 2014;289(7):3913-22.

28. Pertschy B, Zisser G, Schein H, Köffel R, Rauch G, Grillitsch K, et al. Diazaborine treatment of yeast cells inhibits maturation of the 605 ribosomal subunit. Mol Cell Biol. 2004;24(14):6476-87.

29. Zisser G, Ohmayer U, Mauerhofer C, Mitterer V, Klein I, Rechberger GN, et al. Viewing pre-60S maturation at a minute's timescale. Nucleic Acids Res. 2018;46(6):3140-51.

30. Kawashima SA, Chen Z, Aoi Y, Patgiri A, Kobayashi Y, Nurse P, et al. Potent, reversible, and specific chemical inhibitors of eukaryotic ribosome biogenesis. Cell. 2016;167(2):512-524.e14.

31. Ulbrich C, Diepholz M, Baßler J, Kressler D, Pertschy B, Galani K, et al. Mechanochemical removal of ribosome biogenesis factors from nascent 605 ribosomal subunits. Cell. 2009;138(5):911-22.

32. Gadal O, Strauß D, Braspenning J, Hoepfner D, Petfalski E, Philippsen $P$, et al. A nuclear AAA-type ATPase (Rix7p) is required for biogenesis and nuclear export of 605 ribosomal subunits. EMBO J. 2001:20(14): 3695-704.

33. Ban N, Beckmann R, Cate JH, Dinman JD, Dragon F, Ellis SR, et al. A new system for naming ribosomal proteins. Curr Opin Struct Biol. 2014; 24:165-9.

34. Chen W, Xie Z, Yang F, Ye K. Stepwise assembly of the earliest precursors of large ribosomal subunits in yeast. Nucleic Acids Res. 2017;45(11):6837-47.

35. Gamalinda M, Ohmayer U, Jakovljevic J, Kumcuoglu B, Woolford J, Mbom B, et al. A hierarchical model for assembly of eukaryotic 605 ribosomal subunit domains. Genes Dev. 2014;28(2):198-210.

36. Jakovljevic J, Ohmayer U, Gamalinda M, Talkish J, Alexander L, Linnemann J, et al. Ribosomal proteins L7 and L8 function in concert with six A3 assembly factors to propagate assembly of domains I and II of 25S rRNA in yeast 60 s ribosomal subunits. RNA. 2012;18(10):1805-22.

37. Ohmayer U, Gamalinda M, Sauert M, Ossowski J, Pöll G, Linnemann J, et al. Studies on the assembly characteristics of large subunit ribosomal proteins in S. cerevisae. PloS One. 2013;8(7):e68412

38. Ohmayer U, Gil-Hernández Á, Sauert M, Martín-Marcos P, Tamame M, Tschochner $\mathrm{H}$, et al. Studies on the coordination of ribosomal protein assembly events involved in processing and stabilization of yeast early large ribosomal subunit precursors. PLoS One. 2015;10(12):e0143768.

39. Pöll G, Braun $T$, Jakovljevic J, Neueder A, Jakob S, Jr JLW, et al. rRNA maturation in yeast cells depleted of large ribosomal subunit proteins. PLoS One. 2009:4(12):e8249.

40. Barandun J, Chaker-Margot M, Hunziker M, Molloy KR, Chait BT, Klinge S. The complete structure of the small-subunit processome. Nat Struct Mol Biol. 2017. https://doi.org/10.1038/nsmb.3472.
41. Ferreira-Cerca S, Pöll G, Gleizes P-E, Tschochner H, Milkereit P. Roles of eukaryotic ribosomal proteins in maturation and transport of pre-18S rRNA and ribosome function. Mol Cell. 2005;20(2):263-75.

42. Ferreira-Cerca S, Pöll G, Kühn H, Neueder A, Jakob S, Tschochner $H$, et al. Analysis of the in vivo assembly pathway of eukaryotic $40 \mathrm{~S}$ ribosomal proteins. Mol Cell. 2007;28(3):446-57.

43. Kreuzer J, Bach NC, Forler D, Sieber SA. Target discovery of acivicin in cancer cells elucidates its mechanism of growth inhibition. Chem Sci. 2015;6(1):237-45

44. Harris MN, Medrek TJ, Golomb FM, Gumport SL, Postel AH, Wright JC. Chemotherapy with streptonigrin in advanced cancer. Cancer. 1965; 18(1):49-57.

45. Rivers SL, Whittington RM, Medrek TJ. Treatment of malignant lymphomas with methyl ester of streptonigrin (NSC 45384). Cancer. 1966;19(10):1377-85

46. Li X, Xu S, Tan Y, Chen J. The effects of idarubicin versus other anthracyclines for induction therapy of patients with newly diagnosed leukaemia. Cochrane Database Syst Rev. 2015;6:CD010432.

47. Leonard RC, Cameron DA, Anderson A, Ostrowski J, Howell A. Idarubicin and cyclophosphamide--an active oral chemotherapy regimen for advanced breast cancer. Crit Rev Oncol Hematol. 2000;33(1):61-6.

48. Luzina OA, Salakhutdinov NF. Biological activity of usnic acid and its derivatives: part 2. Effects on higher organisms. Molecular and physicochemical aspects. Russ J Bioorganic Chem. 2016:42(3):249-68.

49. Luzina OA, Salakhutdinov NF. Usnic acid and its derivatives for pharmaceutical use: a patent review (2000-2017). Expert Opin Ther Pat. 2018:28(6):477-91.

50. Sivanandam A, Murthy S, Chinnakannu K, Bai U, Kim S-H, Barrack ER, et al. Calmodulin protects androgen receptor from CALPAIN-mediated breakdown in prostate cancer cells. J Cell Physiol. 2011;226(7):1889-96.

51. Sack U, Walther W, Scudiero D, Selby M, Aumann J, Lemos C, et al. S100A4induced cell motility and metastasis is restricted by the Wnt/ $\beta$-catenin pathway inhibitor calcimycin in colon cancer cells. Mol Biol Cell. 2011;22(18): 3344-54.

52. Devassy JG, Nwachukwu ID, Jones PJH. Curcumin and cancer: barriers to obtaining a health claim. Nutr Rev. 2015;73(3):155-65.

53. Lv C, Zeng H-W, Wang J-X, Yuan X, Zhang C, Fang T, et al. The antitumor natural product tanshinone IIA inhibits protein kinase $C$ and acts synergistically with 17-AAG. Cell Death Dis. 2018:9(2):165.

54. Lin C-Y, Chang T-W, Hsieh W-H, Hung M-C, Lin I-H, Lai S-C, et al. Simultaneous induction of apoptosis and necroptosis by Tanshinone IIA in human hepatocellular carcinoma HepG2 cells. Cell Death Discov. 2016;2:16065

55. Chiu SC, Huang SY, Chen SP, Su CC, Chiu TL, Pang CY. Tanshinone IIA inhibits human prostate cancer cells growth by induction of endoplasmic reticulum stress in vitro and in vivo. Prostate Cancer Prostatic Dis. 2013;16(4):315-22.

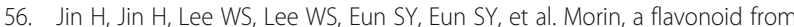
Moraceae, suppresses growth and invasion of the highly metastatic breast cancer cell line MDA-MB-231 partly through suppression of the Akt pathway. Int J Oncol. 2014;45(4):1629-37.

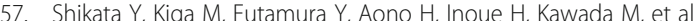
Mitochondrial uncoupler exerts a synthetic lethal effect against $\beta$-catenin mutant tumor cells. Cancer Sci. 2017:108(4):772-84.

58. Diogo CV, Machado NG, Barbosa IA, Serafim TL, Burgeiro A, Oliveira PJ. Berberine as a promising safe anti-cancer agent - is there a role for mitochondria? Curr Drug Targets. 2011;12(6):850-9.

59. Chen J, Stubbe J. Bleomycins: towards better therapeutics. Nat Rev Cancer. 2005;5(2):102-12.

60. Watanabe M, Kodaira S, Takahashi T, Tominaga T, Hojo K, Kato T, et al. Randomized trial of the efficacy of adjuvant chemotherapy for colon cancer with combination therapy incorporating the oral pyrimidine 1-hexylcarbamoyl5-fluorouracil. Langenbeck's Arch Surg. 2006;391(4):330-7.

61. Kubota T, Fujita S, Kodaira S, Yamamoto T, Josui K, Arisawa Y, et al. Antitumor activity of fluoropyrimidines and thymidylate synthetase inhibition. Jpn J Cancer Res Gann. 1991;82(4):476-82.

62. Shelton J, Lu X, Hollenbaugh JA, Cho JH, Amblard F, Schinazi RF. Metabolism, biochemical actions, and chemical synthesis of anticancer nucleosides, nucleotides, and base analogs. Chem Rev. 2016:116(23):14379-455.

63. Kilic N, Aras S, Cansaran-Duman D. Determination of vulpinic acid effect on apoptosis and mRNA expression levels in breast cancer cell lines. Anticancer Agents Med Chem. 2018;18(14):2032-41. 
64. Sweeney MJ. Mycophenolic acid and its mechanism of action in cancer and psoriasis. Jpn J Antibiot. 1977;30(Suppl):85-92.

65. Dun B, Sharma A, Teng Y, Liu H, Purohit S, Xu H, et al. Mycophenolic acid inhibits migration and invasion of gastric cancer cells via multiple molecular pathways. PLoS One. 2013;8(11):e81702.

66. Xiong W, Li W-H, Jiang Y-X, Liu S, Ai Y-Q, Liu R, et al. Parecoxib: an enhancer of radiation therapy for colorectal cancer. Asian Pac J Cancer Prev APJCP. 2015;16(2):627-33.

67. Li L-Y, Xiao J, Liu Q, Xia K. Parecoxib inhibits glioblastoma cell proliferation, migration and invasion by upregulating miRNA-29c. Biol Open. 2017;6(3):311-6.

68. Kalantari K, Moniri M, Boroumand Moghaddam A, Abdul Rahim R, Bin Ariff A, Izadiyan $Z$, et al. A review of the biomedical applications of zerumbone and the techniques for its extraction from ginger rhizomes. Molecules. 2017; 22(10):1645.

69. Woo Y, Jung Y-J. Angiotensin II receptor blockers induce autophagy in prostate cancer cells. Oncol Lett. 2017;13(5):3579-85.

70. Ni X, Hu G, Cai X. The success and the challenge of all-trans retinoic acid in the treatment of cancer. Crit Rev Food Sci Nutr. 2018;0(0):1-10.

71. Aydoğmuş-Öztürk F, Jahan H, Beyazit N, Günaydın K, Choudhary MI. The anticancer activity of visnagin, isolated from Ammi visnaga L., against the human malignant melanoma cell lines, HT 144. Mol Biol Rep. 2019;46(2): 1709-14.

72. Iwasaki T, Mukai M, Tsujimura T, Tatsuta M, Nakamura $H$, Terada N, et al. Ipriflavone inhibits osteolytic bone metastasis of human breast cancer cells in a nude mouse model. Int J Cancer. 2002;100(4):381-7.

73. Čáňová K, Rozkydalová L, Rudolf E. Anthelmintic flubendazole and its potential use in anticancer therapy. Acta Medica Hradec Kralove Czech Repub. 2017:60(1):5-11.

74. Gregory EJ, Cohen SC, Oines DW, Mims CH. Megestrol acetate therapy for advanced breast cancer. J Clin Oncol. 1985:3(2):155-60.

75. Johnson PA, Bonomi PD, Anderson KM, Wolter JM, Economou SG. Megestrol acetate: first-line therapy for advanced breast cancer. Semin Oncol. 1986;13(4 Suppl 4):15-9.

76. Pronzato P, Brema F, Amoroso D, Bertelli G, Conte PF, Martini MC, et al. Megestrol acetate: phase II study of a single daily administration in advanced breast cancer. Breast Cancer Res Treat. 1990;17(1):51-4.

77. Meng Z, Cao R, Yang Z, Liu T, Wang Y, Wang X. Inhibitor of 5-lipoxygenase, zileuton, suppresses prostate cancer metastasis by upregulating E-cadherin and paxillin. Urology. 2013;82(6):1452.e7-1452.e14.

78. Hilf R, Bell C, Goldenberg H, Michel I. Effect of fluphenazine $\mathrm{HC} 1$ on R3230AC mammary carcinoma and mammary glands of the rat. Cancer Res. 1971;31(8):1111-7.

79. Kuzu OF, Gowda R, Noory MA, Robertson GP. Modulating cancer cell survival by targeting intracellular cholesterol transport. Br J Cancer. 2017; 117(4):513-24.

80. Happy M, Dejoie J, Zajac CK, Cortez B, Chakraborty K, Aderemi J, et al. Sigma 1 receptor antagonist potentiates the anti-cancer effect of p53 by regulating ER stress, ROS production, Bax levels, and caspase-3 activation. Biochem Biophys Res Commun. 2015;456(2):683-8.

81. Herzig RH, Lazarus HM, Wolff SN, Phillips GL, Herzig GP. High-dose cytosine arabinoside therapy with and without anthracycline antibiotics for remission reinduction of acute nonlymphoblastic leukemia. J Clin Oncol. 1985;3(7):992-7.

82. Wolff SN, Marion J, Stein RS, Flexner JM, Lazarus HM, Spitzer TR, et al. High-dose cytosine arabinoside and daunorubicin as consolidation therapy for acute nonlymphocytic leukemia in first remission: a pilot study. Blood. 1985;65(6):1407-11.

83. Czaykowski PM, Samuels T, Oza A. A durable response to cytarabine in advanced breast cancer. Clin Oncol. 1997;9(3):181-3.

84. Chen YL, Law PY, Loh HH. Inhibition of Akt/protein kinase B signaling by naltrindole in small cell lung cancer cells. Cancer Res. 2004;64(23):8723-30.

85. Mundra JJ, Terskiy A, Howells RD. Naltrindole inhibits human multiple myeloma cell proliferation in vitro and in a murine xenograft model in vivo. J Pharmacol Exp Ther. 2012;342(2):273-87.

86. Kaya TT, Altun A, Turgut NH, Ataseven H, Koyluoglu G. Effects of a multikinase inhibitor Motesanib (AMG 706) alone and combined with the selective DuP-697 COX-2 inhibitor on colorectal cancer cells. Asian Pac J Cancer Prev. 2016;17(3):1103-10.

87. Sweeney MJ, Boder B, Cullinan GJ, Culp W, Daniels D. Antitumor activity of deacetyl vinblastine amide sulfate (vindesine) in rodents and mitotic accumulation studies in culture, vol. 7; 1978
88. Walker BK, Raich PC, Fontana J, Subramanian VP, Rogers JS, Knost JA, et al Phase II study of vindesine in patients with advanced breast cancer. Cancer Treat Rep. 1982;66(9):1729-32.

89. Hainsworth JD, Porter LL, Johnson DH, Hande KR, Wolff SN, Birch R, et al. Combination chemotherapy with vindesine, etoposide, and cisplatin in nonsmall cell lung cancer: a pilot study of the Southeastern Cancer Study Group. Cancer Treat Rep. 1986;70(3):339-41.

90. Paik WH, Ryu JK, Jeong K-S, Park JM, Song BJ, Lee SH, et al. Clobenpropit enhances anti-tumor effect of gemcitabine in pancreatic cancer. World J Gastroenterol WJG. 2014;20(26):8545-57.

91. Kleinberg DL, Boyd AE, Wardlaw S, Frantz AG, George A, Bryan N, et al. Pergolide for the treatment of pituitary tumors secreting prolactin or growth hormone. N Engl J Med. 1983;309(12):704-9.

92. Hu W, Tian H, Yue W, Li L, Li S, Gao C, et al. Rotenone induces apoptosis in human lung cancer cells by regulating autophagic flux. IUBMB Life. 2016; 68(5):388-93.

93. Deng $\mathrm{Y}-\mathrm{T}$, Huang $\mathrm{H}-\mathrm{C}$, Lin J-K. Rotenone induces apoptosis in MCF-7 human breast cancer cell-mediated ROS through JNK and p38 signaling. Mol Carcinog. 2010;49(2):141-51.

94. Li N, Ragheb K, Lawler G, Sturgis J, Rajwa B, Melendez JA, et al. Mitochondrial complex I inhibitor rotenone induces apoptosis through enhancing mitochondrial reactive oxygen species production. J Biol Chem. 2003;278(10):8516-25

95. Huang F, Wang P, Wang X. Thapsigargin induces apoptosis of prostate cancer through cofilin-1 and paxillin. Oncol Lett. 2018;16(2):1975-80.

96. Wang $X$, Xiong W, Tang Y. Tunicamycin suppresses breast cancer cell growth and metastasis via regulation of the protein kinase $B /$ nuclear factorKB signaling pathway. Oncol Lett. 2018;15(4):4137-42.

97. Zhu P, Wu Y, Yang A, Fu X, Mao M, Liu Z. Catalpol suppressed proliferation, growth and invasion of CT26 colon cancer by inhibiting inflammation and tumor angiogenesis. Biomed Pharmacother. 2017;95:68-76.

98. Bai F, Liu K, Li H, Wang J, Zhu J, Hao P, et al. Veratramine modulates AP-1dependent gene transcription by directly binding to programmable DNA. Nucleic Acids Res. 2018:46(2):546-57.

99. Juarez M, Schcolnik-Cabrera A, Dueñas-Gonzalez A. The multitargeted drug ivermectin: from an antiparasitic agent to a repositioned cancer drug. Am J Cancer Res. 2018:8(2):317-31.

100. Brem SS, Zagzag D, Tsanaclis AM, Gately S, Elkouby MP, Brien SE. Inhibition of angiogenesis and tumor growth in the brain. Suppression of endothelial cell turnover by penicillamine and the depletion of copper, an angiogenic cofactor. Am J Pathol. 1990;137(5):1121-42.

101. Brem S, Grossman SA, Carson KA, New P, Phuphanich S, Alavi JB, et al. Phase 2 trial of copper depletion and penicillamine as antiangiogenesis therapy of glioblastoma. Neuro-Oncol. 2005;7(3):246-53.

102. Wadhwa S, Mumper RJ. D-penicillamine and other low molecular weight thiols: review of anticancer effects and related mechanisms. Cancer Lett. 2013:337(1):8-21.

103. Feli A, Jazayeri S, Bitaraf MA, Dodaran MS, Parastouei $K$, HosseinzadehAttar MJ. Combination therapy with low copper diet, penicillamine and gamma knife radiosurgery reduces VEGF and IL-8 in patients with recurrent glioblastoma. Asian Pac J Cancer Prev APJCP. 2017;18(7):19992003

104. Yu S-T, Chen T-M, Tseng S-Y, Chen Y-H. Tryptanthrin inhibits MDR1 and reverses doxorubicin resistance in breast cancer cells. Biochem Biophys Res Commun. 2007;358(1):79-84.

105. Kimoto T, Hino K, Koya-Miyata S, Yamamoto Y, Takeuchi M, Nishizaki Y, et al. Cell differentiation and apoptosis of monocytic and promyelocytic leukemia cells (U-937 and HL-60) by tryptanthrin, an active ingredient of Polygonum tinctorium Lour. Pathol Int. 2001;51(5):315-25.

106. Chan H-L, Yip H-Y, Mak N-K, Leung K-N. Modulatory effects and action mechanisms of tryptanthrin on murine myeloid leukemia cells. Cell Mo Immunol. 2009:6(5):335-42.

107. Kashyap D, Sharma A, Tuli HS, Sak K, Mukherjee T, Bishayee A. Molecular targets of celastrol in cancer: recent trends and advancements. Crit Rev Oncol Hematol. 2018;128:70-81.

108. López-Lázaro M, de la Peña NP, Pastor N, Martín-Cordero C, Navarro E, Cortés F, et al. Anti-tumour activity of Digitalis purpurea L. subsp. heywoodii. Planta Med. 2003;69(8):701-4.

109. Saleh A, ElFayoumi HM, Youns M, Barakat W. Rutin and orlistat produce antitumor effects via antioxidant and apoptotic actions. Naunyn Schmiedeberg's Arch Pharmacol. 2019;392(2):165-75. 
110. Kridel SJ, Axelrod F, Rozenkrantz N, Smith JW. Orlistat is a novel inhibitor of fatty acid synthase with antitumor activity. Cancer Res. 2004;64(6):2070-5.

111. Lewinska A, Siwak J, Rzeszutek I, Wnuk M. Diosmin induces genotoxicity and apoptosis in DU145 prostate cancer cell line. Toxicol in Vitro. 2015; 29(3):417-25.

112. Kuntz S, Wenzel U, Daniel H. Comparative analysis of the effects of flavonoids on proliferation, cytotoxicity, and apoptosis in human colon cancer cell lines. Eur J Nutr. 1999;38(3):133-42.

113. Liu Z, Ha U-S, Yu K, Wu C, Yokoyama N, Zi X. Kavalactone yangonin induces autophagy and sensitizes bladder cancer cells to flavokawain $\mathrm{A}$ and docetaxel via inhibition of the mTOR pathway. J Biomed Res. 2017;31(5):408-18.

114. Choque E, Schneider C, Gadal O, Dez C. Turnover of aberrant pre-40S preribosomal particles is initiated by a novel endonucleolytic decay pathway. Nucleic Acids Res. 2018;46(9):4699-714.

115. Kos-Braun IC, Jung I, Koš M. Tor1 and CK2 kinases control a switch between alternative ribosome biogenesis pathways in a growth-dependent manner. PLoS Biol. 2017:15(3):e2000245.

116. Venema J, Tollervey D. Ribosome synthesis in Saccharomyces cerevisiae. Annu Rev Genet. 1999:33(1):261-311.

117. Fang F, Hoskins J, Butler JS. 5-Fluorouracil enhances exosome-dependent accumulation of Polyadenylated rRNAs. Mol Cell Biol. 2004:24(24):10766-76.

118. Lum PY, Armour CD, Stepaniants SB, Cavet G, Wolf MK, Butler JS, et al. Discovering modes of action for therapeutic compounds using a genomewide screen of yeast heterozygotes. Cell. 2004;116(1):121-37.

119. Taymaz-Nikerel H, Karabekmez ME, Eraslan S, Kırdar B. Doxorubicin induces an extensive transcriptional and metabolic rewiring in yeast cells. Sci Rep. 2018;8(1):13672.

120. Bolzán AD, Bianchi MS. Genotoxicity of streptonigrin: a review. Mutat Res Mutat Res. 2001;488(1):25-37

121. Dementiev A, Joachimiak A, Nguyen H, Gorelik A, Illes K, Shabani S, et al. Molecular mechanism of inhibition of acid ceramidase by carmofur. J Med Chem. 2019;62(2):987-92.

122. Nguyen HS, Awad AJ, Shabani S, Doan N. Molecular targeting of acid ceramidase in glioblastoma: a review of its role, potential treatment, and challenges. Pharmaceutics. 2018;10(2):45.

123. Realini N, Solorzano C, Pagliuca C, Pizzirani D, Armirotti A, Luciani R, et al. Discovery of highly potent acid ceramidase inhibitors with in vitro tumor chemosensitizing activity. Sci Rep. 2013;3:1035.

124. Sato S, Ueyama T, Fukui H, Miyazaki K, Kuwano M. Anti-tumor effects of carmofur on human 5-FU resistant cells. Gan To Kagaku Ryoho. 1999;26(11): 1613-6.

125. Greenhalgh DA, Parish JH. Effect of 5-fluorouracil combination therapy on RNA processing in human colonic carcinoma cells. Br J Cancer. 1990;61 (3, 3):415-9.

126. Hoskins J, Butler JS. RNA-based 5-fluorouracil toxicity requires the pseudouridylation activity of Cbf5p. Genetics. 2008;179(1):323-30.

127. Kammler S, Lykke-Andersen S, Jensen TH. The RNA exosome component hRrp6 is a target for 5-fluorouracil in human cells. Mol Cancer Res. 2008;6(6):990-5.

128. Longley DB, Harkin DP, Johnston PG. 5-Fluorouracil: mechanisms of action and clinical strategies. Nat Rev Cancer. 2003;3(5):330-8.

129. Ghoshal K, Jacob ST. An alternative molecular mechanism of action of 5 fluorouracil, a potent anticancer drug. Biochem Pharmacol. 1997;53(11): 1569-75.

130. Silverstein RA, González de Valdivia E, Visa N. The incorporation of 5fluorouracil into RNA affects the ribonucleolytic activity of the exosome subunit Rrp6. Mol Cancer Res MCR. 2011;9(3):332-40.

131. Houalla R, Devaux F, Fatica A, Kufel J, Barrass D, Torchet C, et al. Microarray detection of novel nuclear RNA substrates for the exosome. Yeast. 2006; 23(6):439-54.

132. Shen M, Wu M-Y, Chen L-P, Zhi Q, Gong F-R, Chen K, et al. Cantharidin represses invasion of pancreatic cancer cells through accelerated degradation of MMP2 mRNA. Sci Rep. 2015;5:11836.

133. Frederick CA, Williams LD, Ughetto $G$, van der Marel GA, van Boom JH, Rich A, et al. Structural comparison of anticancer drug-DNA complexes: adriamycin and daunomycin. Biochemistry. 1990;29(10):2538-49.

134. Nitiss JL. Targeting DNA topoisomerase II in cancer chemotherapy. Nat Rev Cancer. 2009;9(5):338-50

135. Pérez-Arnaiz C, Busto N, Leal JM, García B. New insights into the mechanism of the DNA/doxorubicin interaction. J Phys Chem B. 2014;118(5):1288-95.

136. Yang F, Teves SS, Kemp CJ, Henikoff S. Doxorubicin, DNA torsion, and chromatin dynamics. Biochim Biophys Acta. 2014;1845(1):84-9.
137. Kwon Y, Cha J, Chiang J, Tran G, Giaever G, Nislow C, et al. A chemogenomic approach to understand the antifungal action of Lichenderived vulpinic acid. J Appl Microbiol. 2016;121(6):1580-91.

138. Granneman S, Petfalski E, Tollervey D. A cluster of ribosome synthesis factors regulate pre-rRNA folding and 5.8S rRNA maturation by the Rat1 exonuclease. EMBO J. 2011;30(19):4006-19.

139. Baßler J, Kallas M, Pertschy B, Ulbrich C, Thoms M, Hurt E. The AAA-ATPase Rea1 drives removal of biogenesis factors during multiple stages of 605 ribosome assembly. Mol Cell. 2010;38(5):712-21.

140. Miles TD, Jakovljevic J, Horsey EW, Harnpicharnchai P, Tang L, Woolford JL. Ytm1, Nop7, and Erb1 form a complex necessary for maturation of yeast 665 preribosomes. Mol Cell Biol. 2005;25(23):10419-32.

141. Horigome C, Okada T, Matsuki K, Mizuta K. A ribosome assembly factor Ebp2p, the yeast homolog of EBNA1-binding protein 2, is involved in the secretory response. Biosci Biotechnol Biochem. 2008;72(4):1080-6.

142. Wimalasena TT, Enjalbert B, Guillemette T, Plumridge A, Budge S, Yin Z, et al. Impact of the unfolded protein response upon genome-wide expression patterns, and the role of $\mathrm{Hacl}$ in the polarized growth, of Candida albicans. Fungal Genet Biol. 2008;45(9):1235-47.

143. Yabuki Y, Katayama M, Kodama Y, Sakamoto A, Yatsuhashi A, Funato K, et al. Arp2/3 complex and Mps3 are required for regulation of ribosome biosynthesis in the secretory stress response. Yeast. 2017:34(4):155-63.

144. Rajagopalan PTR, Zhang Z, McCourt L, Dwyer M, Benkovic SJ, Hammes GG. Interaction of dihydrofolate reductase with methotrexate: ensemble and single-molecule kinetics. Proc Natl Acad Sci. 2002;99(21):13481-6.

145. Albrecht LV, Bui MH, Robertis EMD. Canonical Wnt is inhibited by targeting one-carbon metabolism through methotrexate or methionine deprivation. Proc Natl Acad Sci. 2019;116(8):2987-95.

146. Wang Y-C, Chiang E-PI. Low-dose methotrexate inhibits methionine Sadenosyltransferase in vitro and in vivo. Mol Med. 2011;18(1):423-32.

147. Longtine MS, Mckenzie A III, Demarini DJ, Shah NG, Wach A, Brachat A, et al Additional modules for versatile and economical PCR-based gene deletion and modification in Saccharomyces cerevisiae. Yeast. 1998;14(10):953-61.

148. Thomas BJ, Rothstein R. Elevated recombination rates in transcriptionally active DNA. Cell. 1989;56(4):619-30

149. Sturn A, Quackenbusch J, Trajanoski Z. Genesis: cluster analysis of microarray data. Bioinformatics. 2002;1:207-8.

150. Lam YW, Lamond Al, Mann M, Andersen JS. Analysis of nucleolar protein dynamics reveals the nuclear degradation of ribosomal proteins. Curr Biol. 2007;17(9):749-60.

\section{Ready to submit your research? Choose BMC and benefit from:}

- fast, convenient online submission

- thorough peer review by experienced researchers in your field

- rapid publication on acceptance

- support for research data, including large and complex data types

- gold Open Access which fosters wider collaboration and increased citations

- maximum visibility for your research: over $100 \mathrm{M}$ website views per year

At $\mathrm{BMC}$, research is always in progress.

Learn more biomedcentral.com/submission 